\title{
Exploring top quark FCNC within 2HDM type III in association with flavor physics
}

\author{
C.S. Kim, ${ }^{a}$ Yeo Woong Yoon ${ }^{b, c}$ and Xing-Bo Yuan ${ }^{a}$ \\ ${ }^{a}$ Department of Physics and IPAP, \\ Yonsei University, Seoul 120-749, Korea \\ ${ }^{b}$ School of Physics, KonKuk University, \\ Seoul 143-701, Korea \\ ${ }^{c}$ School of Physics, Korea Institute for Advanced Study, \\ Seoul 130-722, Korea \\ E-mail: cskim@yonsei.ac.kr, ywyoon@kias.re.kr, xbyuan@yonsei.ac.kr
}

ABSTRACT: The top quark flavor changing neutral current (FCNC) process is an excellent probe to search for new physics in top sector since the Standard Model expectation is extremely suppressed. We explore Higgs-mediated top quark FCNC, focusing on $H$-t$c$ Yukawa coupling $\lambda_{c t}$ within the general two Higgs doublet model. After electroweak symmetry breaking the top quark FCNC couplings are included in the charged Higgs Yukawa sector so that they contribute to various processes in flavor physics. To probe $\lambda_{c t}$, we study anomalous single top production and the same sign top pair production at the LHC in association with flavor physics from the tree-level processes $B \rightarrow D^{(*)} \tau \nu, B \rightarrow \tau \nu$ as well as from the loop-level processes $B_{d} \rightarrow X_{s} \gamma, B_{d, s}-\bar{B}_{d, s}$ mixing. We perform combined analysis of all the constraints regarding the fine-tuning argument to fit the data and discuss future prospect. The recently updated measurements on $B \rightarrow D^{(*)} \tau \nu$ still prefer large $\lambda_{c t}$, but we show that the current bound on the same sign top pair production at the LHC gives the most significant upper bound on $\lambda_{c t}$ to be less than $10 \sim 30$ depending on neutral heavy Higgs masses. We also find that for the given upper bound on $\lambda_{c t}, B \rightarrow D^{(*)} \tau \nu$ put significant lower bound on $H-\tau-\tau$ Yukawa coupling, and the bound is proportional to the charged Higgs mass.

Keywords: Higgs Physics, Beyond Standard Model, B-Physics, Rare Decays

ARXiv EPrint: 1509.00491 


\section{Contents}

1 Introduction 1

2 Yukawa sector of aligned 2HDM type III 3

3 Method of numerical analysis 4

4 Top quark FCNC processes at colliders 5

$4.1 t \rightarrow c g \quad 7$

$4.2 \quad c c \rightarrow t t$

5 Flavor physics - Tree-level processes 9

6 Flavor physics - Loop-level processes 12

$\begin{array}{lll}6.1 & B_{d} \rightarrow X_{s} \gamma & 12\end{array}$

$6.2 B_{d, s}-\bar{B}_{d, s}$ mixing 13

7 Combined analysis and future prospect 16

8 Conclusion $\quad 19$

A Form factors in $t \rightarrow c g \quad 20$

\section{Introduction}

The top quark, the heaviest particle in the Standard Model (SM), plays an important role as an input for the electroweak (EW) precision measurements [1]. Because its mass is much heavier than other known particles, the top quark is considered to be the most viable candidate which has a close connection to new physics (NP) that controls the EW symmetry breaking mechanism. Meanwhile, the discovery of the SM-like Higgs boson at the LHC [2,3] and the precision measurement of its property $[4,5]$ shed much light on the physics in EW sector, boosting the relevant studies. Especially, NP scenarios with extended Higgs sector have received great interest due to its rich phenomenology and attempt to complement the SM $[6,7]$.

One of the simplest scenarios with extended Higgs sector is to introduce a new Higgs doublet. Because the two Higgs doublets can couple to both up-type and down-type quarks, after rotating into their mass eigenstates, the tree-level flavor changing neutral current (FCNC) inevitably arises. In the SM, the tree-level FCNC is forbidden by the GIM mechanism [8]. The FCNC process only takes place through the loop diagrams with charged 
current and rough estimation of the loop correction at the amplitude level is

$$
V_{\mathrm{CKM}}^{\prime} V_{\mathrm{CKM}}^{*} \frac{\alpha_{e}}{4 \pi}\left(\frac{m_{q}}{m_{W}}\right)^{2},
$$

where $V_{\mathrm{CKM}}^{(\prime)}$ are CKM matrices, $m_{q}$ is the mass of quark inside the loop. Thus, the loopinduced down-type quark FCNC processes such as $b \rightarrow s \gamma$, which is involved with top quark loop, has enhancement factor $\left(m_{t} / m_{W}\right)^{2}$ and their rates mostly fall within current experimental reach of $B$ physics and Kaon physics. Therefore, the down-type quark FCNC is severely constrained and dangerous to many NP scenarios. On the other hand, the uptype quark FCNC processes, for example top quark FCNC process $t \rightarrow c \gamma$, are involved with $b$-quark loop and extremely suppressed by $\left(m_{b} / m_{W}\right)^{2}$. The estimation of $\mathcal{B}(t \rightarrow c \gamma)$ is $\mathcal{O}\left(10^{-12}\right)$ [9] within the $\mathrm{SM}$, far too much behind the current experimental reach.

In order to avoid tree-level FCNC, one usually introduces a discrete $Z_{2}$ symmetry to make each up-type or down-type quark couple to only one Higgs doublet. In the Minimal Supersymmetric Standard Model (MSSM) the supersymmetry itself plays the role. Without such a $Z_{2}$ symmetry, the general $2 \mathrm{HDM}$ which is called "2HDM type III" follows a specific scheme to circumvent severe down-type quark FCNC constraints such as the natural flavor conservation [10], the minimal flavor violation [11-16] and Cheng-Sher ansatz [17]. In this work we adopt the last one, in which the Yukawa coupling $\xi_{i j}$ connecting quarks with flavor indices $i, j$ to one of the neutral Higgses is described as

$$
\xi_{i j}=\lambda_{i j} \frac{\sqrt{2 m_{i} m_{j}}}{v},
$$

where $v$ is the $\mathrm{SM}$ vacuum expectation value (vev), $v=246 \mathrm{GeV}, \lambda_{i j}$ is considered to be $\mathcal{O}(1)$. With this ansatz, down-type quark FCNC is severely suppressed due to the small masses of $u, d, s$ quarks, being safe against the experimental constraints. However, top quark FCNC process can be potentially large and should be explored in collider physics as well as in flavor physics.

In the 2HDM type III, after EW symmetry breaking the top quark FCNC Yukawa couplings $\lambda_{q t}(q=u, c)$ also come into play in charged Higgs Yukawa couplings. Therefore, the phenomenology of top quark FCNC process with neutral Higgs exchange is naturally in connection with flavor physics process with charged Higgs exchanged due to the common Yukawa couplings $\lambda_{q t}$. Studies on the top quark FCNC in collider physics especially through anomalous top quark decays were performed in refs. [18-22]. There have been studies on the issue that large top quark FCNC coupling $\lambda_{c t}$ is needed $[23,24]$ to explain the measurements of $\mathcal{B}\left(B_{d} \rightarrow D^{(*)} \tau \nu\right)$ at BaBar [25], which were quite larger than the SM expectations. The authors of ref. [26] study the collider signature with constraints from $b \rightarrow s \gamma$ concerning the perturbativity of Yukawa couplings within the 2HDM and the MSSM. For more comprehensive study on 2HDM type III contribution to both collider and flavor physics, we refer to ref. [27]. The model independent approach using low energy effective operators was done in ref. [28].

In this work we focus on $H$-t-c FCNC coupling $\lambda_{c t}$ within 2HDM type III by adopting Cheng-Sher ansatz. We perform detailed study on several experimental observables that 
can give bound on $\lambda_{c t}$ from collider physics and flavor physics with the most up-to-date experimental data. The issue on $\mathcal{B}\left(B_{d} \rightarrow D^{(*)} \tau \nu\right)$ is revisited with new data from Belle and LHCb. Especially it will be shown that the search for the same sign top pair production at the LHC plays crucial role to constrain $\lambda_{c t}$. Since the current precision measurements of the SM Higgs properties are very well consistent with the SM expectations $[4,5]$, we assume the alignment limit for the Higgs potential of 2HDM type III, in which the SM Higgs sector is well decoupled from the NP sector.

The paper is organized as follows. In section 2, we briefly describe and discuss about the Yukawa structure of aligned 2HDM type III. Section 3 explains about the method of numerical analysis in this work. In section 4, we study the top quark FCNC processes and investigate the bounds from the LHC experiment. In section 5 and 6 , we study the constraints from the flavor physics with tree-level and loop-level processes. Section 7 is reserved for the combined analysis and future prospect for the constraints on $\lambda_{c t}$. We conclude and summarize our result in section 8.

\section{Yukawa sector of aligned 2HDM type III}

The Yukawa interaction Lagrangian of 2HDM type III can be described as [29]

$$
-\mathcal{L}_{\mathrm{Y}}=\bar{Q}_{L}\left(Y_{1}^{d} \Phi_{1}+Y_{2}^{d} \Phi_{2}\right) d_{R}+\bar{Q}_{L}\left(Y_{1}^{u} \tilde{\Phi}_{1}+Y_{2}^{u} \tilde{\Phi}_{2}\right) u_{R}+\bar{L}_{L}\left(Y_{1}^{\ell} \Phi_{1}+Y_{2}^{\ell} \Phi_{2}\right) e_{R}+\text { h.c. }
$$

where $Q_{L}, L_{L}$ are left-handed quark and lepton doublets while $u_{R}, d_{R}, e_{R}$ are right-handed singlets in interaction basis. The two Higgs doublets $\Phi_{1}$ and $\Phi_{2}$ are introduced with the definition $\tilde{\Phi}_{i}=i \sigma_{2} \Phi_{i}^{*}$ where $\sigma_{2}$ is Pauli matrix. $Y_{1,2}^{u, d, \ell}$ are corresponding Yukawa matrices where the flavor indices are implicitly considered. After the EW symmetry breaking $\Phi_{1}$ and $\Phi_{2}$ have the vevs $\left\langle\Phi_{i}\right\rangle=v_{i} / \sqrt{2}$ which satisfies $v_{1}^{2}+v_{2}^{2}=v^{2}$, where $v=246 \mathrm{GeV}$. As usual, we define $\tan \beta=v_{2} / v_{1}$.

Then, we diagonalize mass matrices for fermions from eq. (2.1) and for Higgses from Higgs potential Lagrangian which is described in many literatures (We refer to review paper ref. [7]). We define $\alpha$ as a mixing angle of neutral CP-even Higgses. As we discussed in the introduction, we adopt the alignment limit that specifies

$$
\sin (\beta-\alpha)=1
$$

to make the model comply with the Higgs precision measurement [30-37]. With this alignment limit, the Yukawa Lagrangian eq. (2.1) is re-expressed in terms of mass eigenstates as follows

$$
\begin{aligned}
\mathcal{L}_{\mathrm{Y}}= & \mathcal{L}_{\mathrm{Y}, \mathrm{SM}}+\frac{1}{\sqrt{2}} \bar{d} \xi^{d} d H+\frac{1}{\sqrt{2}} \bar{u} \xi^{u} u H+\frac{1}{\sqrt{2}} \bar{\ell}^{\ell} \ell H-\frac{i}{\sqrt{2}} \bar{d} \gamma_{5} \xi^{d} d A-\frac{i}{\sqrt{2}} \bar{u} \gamma_{5} \xi^{u} u A \\
& -\frac{i}{\sqrt{2}} \bar{\ell} \gamma_{5} \xi^{\ell} \ell A+\left[\bar{u}\left(\xi^{u} V_{\mathrm{CKM}} P_{L}-V_{\mathrm{CKM}} \xi^{d} P_{R}\right) d H^{+}-\bar{\nu} \xi^{\ell} P_{R} \ell H^{+}+\text {h.c. }\right]
\end{aligned}
$$

by ignoring Goldstone Lagrangian. Here, $\mathcal{L}_{\mathrm{Y}, \mathrm{SM}}$ is equal to the SM Yukawa Lagrangian, $u, d, \ell$ are mass eigenstates of up- and down-type quarks and leptons, $H, A$ are $\mathrm{CP}$-even and -odd neutral Higgses, and $H^{ \pm}$are charged Higgses. $V_{\mathrm{CKM}}$ is the CKM matrix, $P_{L}$ and 


\begin{tabular}{|lll|}
\hline$\left|V_{u s}\right| f_{+}^{K \rightarrow \pi}(0)$ & $0.21664 \pm 0.00048$ & {$[38]$} \\
$\left|V_{u b}\right|$ (semi-leptonic) & $(3.70 \pm 0.12 \pm 0.26) \times 10^{-3}$ & {$[38]$} \\
$\left|V_{c b}\right|$ (semi-leptonic) & $(41.0 \pm 0.33 \pm 0.74) \times 10^{-3}$ & {$[38]$} \\
$\left.\gamma^{\circ}\right]$ & $73.2_{-7.0}^{+6.3}$ & {$[38]$} \\
\hline $\bar{m}_{c}\left(\bar{m}_{c}\right)$ & $(1.286 \pm 0.013 \pm 0.040) \mathrm{GeV}$ & {$[38]$} \\
$\bar{m}_{b}\left(\bar{m}_{b}\right)$ & $(4.18 \pm 0.03) \mathrm{GeV}$ & {$[58]$} \\
$\bar{m}_{t}\left(\bar{m}_{t}\right)$ & $(165.95 \pm 0.35 \pm 0.64) \mathrm{GeV}$ & {$[38]$} \\
\hline$f_{+}^{K \rightarrow \pi}(0)$ & $0.9641 \pm 0.0015 \pm 0.0045$ & {$[38]$} \\
$f_{B_{s}}$ & $(225.6 \pm 1.1 \pm 5.4) \mathrm{MeV}$ & {$[38]$} \\
$f_{B_{s}} / f_{B_{d}}$ & $1.205 \pm 0.004 \pm 0.007$ & {$[38]$} \\
$\hat{B}_{B_{s}}$ & $1.320 \pm 0.017 \pm 0.030$ & {$[38]$} \\
$\hat{B}_{B_{s}} / \hat{B}_{B_{d}}$ & $1.023 \pm 0.013 \pm 0.014$ & {$[38]$} \\
\hline
\end{tabular}

Table 1. The theoretical input parameters used in the numerical analysis.

$P_{R}$ are chiral projection operators, $P_{L, R}=\frac{1}{2}\left(1 \mp \gamma_{5}\right)$. Note that in the alignment limit, the SM Yukawa sector is completely decoupled from the NP sector. $\xi^{u, d, \ell}$ are Yukawa matrices for the mass eigenstates which include all the FCNC couplings.

In this work we assume that the new Yukawa matrices are CP-conserving, that is $\xi^{u, d, \ell}$ are real and symmetric:

$$
\xi_{i j}^{u, d, \ell}=\xi_{i j}^{u, d, \ell *}=\xi_{j i}^{u, d, \ell} .
$$

To avoid severe constraints from down-type quark FCNC, we adopt Cheng-Sher ansatz, Eq. (1.2). Due to the tiny masses of $u, d, s$ quarks, the elements of Yukawa couplings that contain those quarks are negligibly small:

$$
\xi^{d} \simeq\left(\begin{array}{ccc}
0 & 0 & 0 \\
0 & 0 & \xi_{s b} \\
0 & \xi_{s b} & \xi_{b b}
\end{array}\right), \quad \xi^{u} \simeq\left(\begin{array}{ccc}
0 & 0 & 0 \\
0 & \xi_{c c} & \xi_{c t} \\
0 & \xi_{c t} & \xi_{t t}
\end{array}\right)
$$

Here, we include $\xi_{s b}$ since it can play some role in our study. In this set-up, the only relevant top-quark FCNC coupling is $\lambda_{c t}$ where $\xi_{c t}=\lambda_{c t} \sqrt{2 m_{c} m_{t}} / v$. It should be emphasized that the top quark FCNC coupling $\lambda_{c t}$ not only belongs to neutral Higgs Yukawa sector but also comes into play in charged Higgs Yukawa sector as can be seen in eq. (2.3). This important feature leads us to probe $\lambda_{c t}$ with the combined analysis of phenomenologies of both collider physics via neutral Higgs exchange and flavor physics via charged Higgs exchange.

\section{$3 \quad$ Method of numerical analysis}

Before we discuss the phenomenology of top quark FCNC, we first summarize theoretical input parameters as well as experimental values that are used in this work and discuss about 
the details of numerical analysis. Table 1 shows input parameters for the processes in flavor physics. The values are taken from the latest result of CKMfitter collaboration [39]. To obtain the uncertainties of theory prediction, we vary each parameter value within $1 \sigma$ range and add each individual uncertainty in quadrature.

In table 2 we summarize experimental data and their SM predictions by using the input values in table 1 . We note that all the SM predictions are in good agreement with the current experimental data, except the ratio $R\left(D^{*}\right)$ which will be discussed in later section. For each observable, the relevant parameters for the theory prediction in 2HDM type III are enumerated. Apparently, those parameters will be constrained by corresponding experimental data. The detailed discussions are presented in the following sections.

As discussed in the previous sections, the relevant model parameters we are interested in aligned 2HDM type III include three mass parameters $M_{H^{ \pm}}, M_{H}, M_{A}$, and four Yukawa couplings $\lambda_{\tau \tau}, \lambda_{b b}, \lambda_{t t}$, and $\lambda_{c t}$. Here, we choose the light neutral Higgs boson $h$ as the observed Higgs boson at the LHC and adopt the alignment limit [40-43]. For other choice that the heavy neutral Higgs $H$ is observed one, we refer to ref. [44, 45]. Direct searches for charged Higgs bosons have been performed at LEP [46], Tevatron [47, 48] and LHC $[49,50]$. The LEP Collaboration put the lower bound $M_{H^{ \pm}} \geq 79.3 \mathrm{GeV}$ by assuming $\mathcal{B}\left(H^{+} \rightarrow \tau^{+} \nu\right)+\mathcal{B}\left(H^{+} \rightarrow c \bar{s}\right)=1$ within $2 \mathrm{HDM}[46]$. The neutral Higgs search at the LEP experiment also put lower bound on the neutral Higgs masses such as $M_{H}>92.8 \mathrm{GeV}$ and $M_{A}>93.4 \mathrm{GeV}$ within CP-conserving MSSM scenario [51]. We adopt those lower limits for heavy Higgs masses as reference values even though above results may depend on Yukawa structure and $m_{\text {SUSY }}$ scale. Indeed, the lower limits of Higgs masses are irrelevant to our main result. With all these considerations, we restrict the parameters of $2 \mathrm{HDM}$ type III in the following ranges:

$$
\begin{aligned}
M_{H^{ \pm}} & \in[80,1000] \mathrm{GeV}, \\
M_{H}\left(M_{A}\right) & \in[125(93), 1000] \mathrm{GeV} .
\end{aligned}
$$

These choices of parameter regions are shown to be reasonable in later section.

In order to derive an allowed parameter space, we impose the experimental constraints in the same way as in refs. [42, 52]: for each point in the theoretical parameter space we span the range of the theory prediction for an observable by performing the $2 \sigma$ variations of input parameters. If the difference between the central values of theory prediction and experimental value is less then the sum of two errors in quadrature, then this point is regarded as allowed. Since the main theoretical uncertainties are due to the hadronic input parameters, common to both the SM and the $2 \mathrm{HDM}$, the relative theoretical uncertainty is assumed to be constant at each point in the parameter space.

\section{Top quark FCNC processes at colliders}

The LHC is often called top-factory since the top pair is copiously produced through QCD interaction. The LHC Run I data already collected millions of top pair events, and even much more top pair events are expected to be collected in the LHC Run II. Undoubtedly, 


\begin{tabular}{|lllll|}
\hline observable & SM & EXP & Ref. & 2HDM parameters \\
\hline $\mathcal{B}(B \rightarrow \tau \nu) \cdot 10^{4}$ & $0.85 \pm 0.14$ & $1.14 \pm 0.22$ & {$[53]$} & $\lambda_{b b}, \lambda_{b s}, \lambda_{b d}, \lambda_{u t}, \lambda_{\tau \tau}, M_{H^{ \pm}}$ \\
$R(D)$ & $0.297 \pm 0.017$ & $0.391 \pm 0.041 \pm 0.028$ & {$[54]$} & $\left(\lambda_{b b}\right), \lambda_{\tau \tau}, \lambda_{c t}, M_{H^{ \pm}}$ \\
$R\left(D^{*}\right)$ & $0.252 \pm 0.003$ & $0.322 \pm 0.018 \pm 0.012$ & {$[54]$} & $\left(\lambda_{b b}\right), \lambda_{\tau \tau}, \lambda_{c t}, M_{H^{ \pm}}$ \\
\hline$\Delta m_{d}\left[\mathrm{ps}^{-1}\right]$ & $0.51 \pm 0.06$ & $0.510 \pm 0.003$ & {$[53]$} & $\left(\lambda_{b b}\right), \lambda_{t t}, \lambda_{c t}, M_{H^{ \pm}}$ \\
$\Delta m_{s}\left[\mathrm{ps}^{-1}\right]$ & $16.93 \pm 1.16$ & $17.757 \pm 0.021$ & {$[53]$} & $\left(\lambda_{b b}\right), \lambda_{b s}, \lambda_{t t}, \lambda_{c t}, M_{H^{ \pm}}$ \\
$\mathcal{B}\left(B \rightarrow X_{s} \gamma\right) \cdot 10^{4}$ & $3.36 \pm 0.23$ & $3.43 \pm 0.22$ & {$[53]$} & $\lambda_{b b}, \lambda_{t t}, \lambda_{c t}, M_{H^{ \pm}}$ \\
\hline $\mathcal{B}(t \rightarrow c g)$ & $<10^{-10}$ & $<1.6 \times 10^{-4}(95 \% \mathrm{CL})$ & {$[55]$} & $\left.\left(\lambda_{b b}\right), \lambda_{t t}, \lambda_{c t}, M_{H^{ \pm}}\right), M_{H}, M_{A}$ \\
$\sigma(p p \rightarrow t t)$ & - & $<62 \mathrm{fb}(95 \% \mathrm{CL})$ & {$[56]$} & $\lambda_{c t}, M_{H}, M_{A}$ \\
$R_{b}$ & $0.21576 \pm 0.00003$ & $0.21629 \pm 0.00066$ & {$[57]$} & $\left(\lambda_{b b}\right), \lambda_{t t}, \lambda_{c t}, M_{H^{ \pm}}$ \\
$\rho_{0}$ & 1 & $1.00040 \pm 0.00024$ & {$[58]$} & $M_{H^{ \pm}}, M_{H}, M_{A}$ \\
\hline
\end{tabular}

Table 2. SM predictions and experimental measurements for the observables used in the numerical analysis. The last column denotes their dependence on the 2HDM parameters. The parameters in the parenthesis imply that they can be safely neglected.

the LHC provides us unique chance to explore the top quark FCNC processes which are extremely small in the SM.

The experimental search for top quark FCNC can be performed either by anomalous decays or production of top quarks at hadron colliders with top quark FCNC couplings [5964]. We note that the searches for $t \rightarrow c h[65,66]$ do not provide any constraints on 2HDM type III in alignment limit since the top quark FCNC couplings with the SM Higgs vanish. The anomalous top decays via $t \rightarrow c / u V$ where $V=\gamma, Z$ are explored at the Tevatron [67-69] and at the LHC [70-73], without finding any significant excess of signal events. However, these searches do not provide any meaningful constraints on 2HDM type III since the prediction is much suppressed by loop correction and EW couplings. Contrary to top decays, the anomalous single top production has much chance to probe top quark FCNC coupling due to the large gluon luminosity in the parton-distribution-function (PDF) and the relatively large QCD coupling. The experimental searches for single top events put upper bound on $\mathcal{B}(t \rightarrow c g)$ and $\mathcal{B}(t \rightarrow u g)$ [74-78]. We focus on $\mathcal{B}(t \rightarrow c g)$ by ignoring $u$-quark involved FCNC process since it is extremely suppressed in Cheng-Sher ansatz even though $u$ quark PDF is bigger than $c$ quark PDF.

The same sign top pair production is a tree-level process and therefore promising to test NP scenarios which contain top quark FCNC couplings. Notable example is that the NP scenario with $Z^{\prime}$ mediated top quark FCNC coupling $[79,80]$ that explains the anomalous top forward-backward asymmetry observed at the Tevatron [81-83] is disfavored by non-observation of the same sign top pair production at the LHC [84, 85]. The recent experimental search at ATLAS with integrated luminosity of $20.3 \mathrm{fb}^{-1}$ at $8 \mathrm{TeV}$ puts the most stringent upper limits on $\sigma(p p \rightarrow t t)$. We interpret the result as an upper limit on $c c \rightarrow t t$ process to constrain $\lambda_{c t}$.

In what follows, we study the phenomenology of $t \rightarrow c g$ and $c c \rightarrow t t$ processes within the 2HDM type III to investigate the top quark FCNC coupling. 


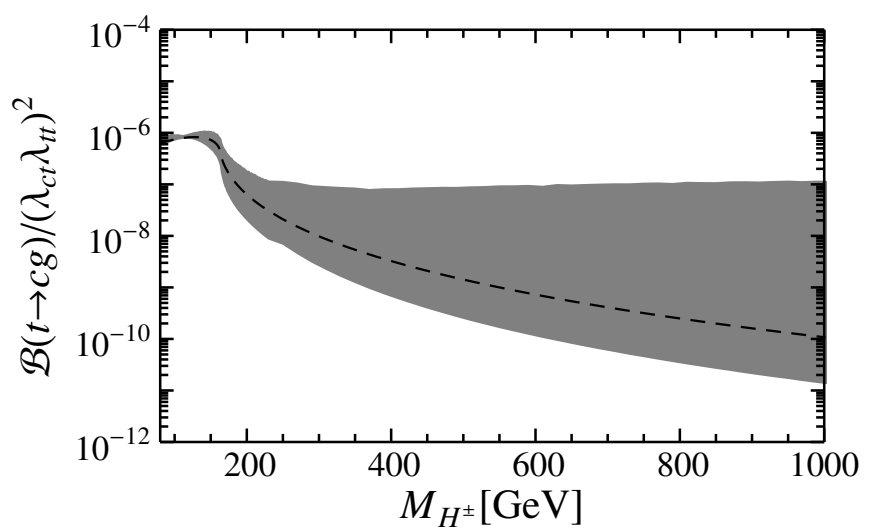

Figure 1. Branching ratio of $t \rightarrow c g$ as a function of the charged Higgs mass. Dashed line: a common scalar mass $M_{H^{ \pm}}=M_{H}=M_{A}$ is taken. Shaded region: neutral Higges' masses $M_{H}$ and $M_{A}$ vary but constrained by the oblique parameter $\Delta \rho$.

\section{$4.1 \quad t \rightarrow c g$}

In the SM, $t \rightarrow c g$ decay is extremely suppressed due to GIM mechanism. However, this rare top decay can be enhanced in some NP scenarios [86, 87]. In general, the form factor for the effective $t c g$ vertex is defined by $[27]^{1}$

$$
\mathcal{L}^{\operatorname{ctg}}=\frac{1}{16 \pi^{2}} \bar{c}\left(\mathcal{A} \gamma^{\mu}+\mathcal{B} \gamma^{\mu} \gamma_{5}+i \mathcal{C} \sigma^{\mu \nu} \frac{q_{\nu}}{m_{t}}+i \mathcal{D} \sigma^{\mu \nu} \frac{q_{\nu}}{m_{t}} \gamma_{5}-\mathcal{A} \frac{m_{t}}{q^{2}} q^{\mu}+\mathcal{B} \frac{m_{t}}{q^{2}} \gamma_{5} q^{\mu}\right) t G_{\mu}^{a} T^{a}
$$

where $T^{a}(a=1, \ldots, 8)$ denote $\mathrm{SU}(3)$ generators. The form factors $\mathcal{A}, \mathcal{B}, \mathcal{C}$ and $\mathcal{D}$ have been calculated in various types of $2 \mathrm{HDM}[9,88,89]$. In the $2 \mathrm{HDM}$ type III, these form factors are generated by the penguin diagrams mediated by the neutral Higgses $h, H$ and $A$ and charged Higgs $H^{ \pm}$. Their explicit expressions are given in appendix A. With the convention eq. (4.1), the decay width for $t \rightarrow c g$ is given by [27]

$$
\Gamma(t \rightarrow c g)=\frac{1}{\left(16 \pi^{2}\right)^{2}} \frac{1}{8 \pi} m_{t} C_{F}\left(|\mathcal{C}|^{2}+|\mathcal{D}|^{2}\right),
$$

with $C_{F}=\left(N_{c}^{2}-1\right) / 2 N_{c}$. We note that $\mathcal{B}(t \rightarrow c g)$ is proportional to $\left(\lambda_{c t} \lambda_{t t}\right)^{2}$ as can be seen from eq. (A.2).

The LHC search for anomalous single top production is performed by ATLAS Collaboration with $14.2 \mathrm{fb}^{-1}$ at $8 \mathrm{TeV}$ [55]. Non-observation of signal put an upper limit on $\mathcal{B}(t \rightarrow c g)$ as

$$
\mathcal{B}(t \rightarrow c g)<1.6 \times 10^{-4} .
$$

In figure 1 we show the plot of $2 \mathrm{HDM}$ type III prediction for $\mathcal{B}(t \rightarrow c g)$ as a function of the charged Higgs mass by setting $\lambda_{c t} \lambda_{t t}=1 .^{2}$ The shaded region is spanned by changing

\footnotetext{
${ }^{1}$ In ref. [27], the last two terms of eq. (4.1) are omitted. Although they do not contribute to the width $\Gamma(t \rightarrow c g)$, they are necessary to satisfy Ward identity.

${ }^{2}$ Our numerical result is consistent with figure 3 of ref. [27] by setting $\xi_{c t}=\xi_{t t}=1$.
} 
neutral Higgses masses under the constraints from $\Delta \rho$. We refer to ref. [43] for detailed analysis of $\Delta \rho$. Even though there can be up to factor $\mathcal{O}\left(10^{3}\right)$ enhancement comparing to the SM expectation for the small $M_{H^{ \pm}}$, the current experimental bound is far above the theory prediction. Therefore, it would be hard to constrain the top quark FCNC parameter space with anomalous single top production measurement at the LHC.

\section{$4.2 \quad c c \rightarrow t t$}

The same sign top pair production at hadron collider requires FCNC coupling with $t-$ or $u$-channel exchange of neutral particle with spin 0 or 1 since the electric charges of final states are same. Another possibility is $s$-channel process mediated by a charge $4 / 3$ new particle. Various NP scenarios that contribute to the same sign top pair production are well summarized in ref. [90] with effective operator formalism. The production rate of the same sign top pair at hadron colliders via the contact interactions with different chiral configuration is modeled in ref. [91]. Meanwhile, in this work we perform the full theory analysis with spin 0 Higgs boson as a mediator since the effective operator formalism may not reproduce well the full theory result if the mediator mass is quite less than $1 \mathrm{TeV}$. We refer to ref. [92] for the analysis with another mediators.

In the 2HDM type III with alignment limit, the same sign top pair production arises at tree level via $t$ - or $u$-channel diagrams with exchange of heavy neutral Higgs bosons, $H$ or $A$. The partonic scattering cross section for $q q \rightarrow t t$ process is described as

$$
\hat{\sigma}(\hat{s})=\int d \hat{t} \frac{1}{64 \pi \hat{s}^{2} N_{c}}\left(\hat{g}_{H}(\hat{s}, \hat{t})+\hat{g}_{A}(\hat{s}, \hat{t})+\hat{g}_{\text {intf }}(\hat{s}, \hat{t})\right)
$$

where the amplitude square functions $\hat{g}_{i}$ are defined as

$$
\begin{aligned}
\hat{g}_{\phi}(\hat{s}, \hat{t}) & =N_{c}^{2} \xi_{c t}^{4}\left[\left(\frac{t-m_{t}^{2}}{t-M_{\phi}^{2}}\right)^{2}+\left(\frac{u-m_{t}^{2}}{u-M_{\phi}^{2}}\right)^{2}+\frac{t u-m_{t}^{2} s-m_{t}^{4}}{N_{c}\left(t-M_{\phi}^{2}\right)\left(u-M_{\phi}^{2}\right)}\right], \\
\hat{g}_{\text {intf }}(\hat{s}, \hat{t}) & =2 N_{c} \xi_{c t}^{4} \frac{\left(t u+m_{t}^{2} s-m_{t}^{4}\right)\left(t u+\left(M_{H}^{2}+M_{A}^{2}\right)\left(s / 2-m_{t}^{2}\right)+M_{H}^{2} M_{A}^{2}\right)}{\left(t-M_{H}^{2}\right)\left(t-M_{A}^{2}\right)\left(u-M_{H}^{2}\right)\left(u-M_{A}^{2}\right)},
\end{aligned}
$$

where $\phi=H, A$. Then the total cross section for $c c \rightarrow t t$ is convoluted with parton luminosity function $f_{c c}\left(x, \mu_{F}\right)$ of sea quark pair $c c$ as follows

$$
\sigma(c c \rightarrow t t)=\int_{\tau}^{1} d x \hat{\sigma}(x s) f_{c c}\left(x, \mu_{F}\right),
$$

where $\tau=4 m_{t}^{2} / s$ and $f_{c c}\left(x, \mu_{F}\right)$ is defined by

$$
f_{c c}\left(x, \mu_{F}\right)=\int_{x}^{1} \frac{d y}{y} f_{c / p}\left(y, \mu_{F}\right) f_{c / p}\left(x / y, \mu_{F}\right) .
$$

Here, $f_{c / p}\left(y, \mu_{F}\right)$ is $c$-quark PDF and the factorization scale $\mu_{F}$ is set to be $\mu_{F}=m_{t}$. We use MSTW2008LO PDF set [93] for the numerical analysis. The gluon and charm quark initial state process with extra jet radiation is not considered by assuming that the contribution is subleading. 

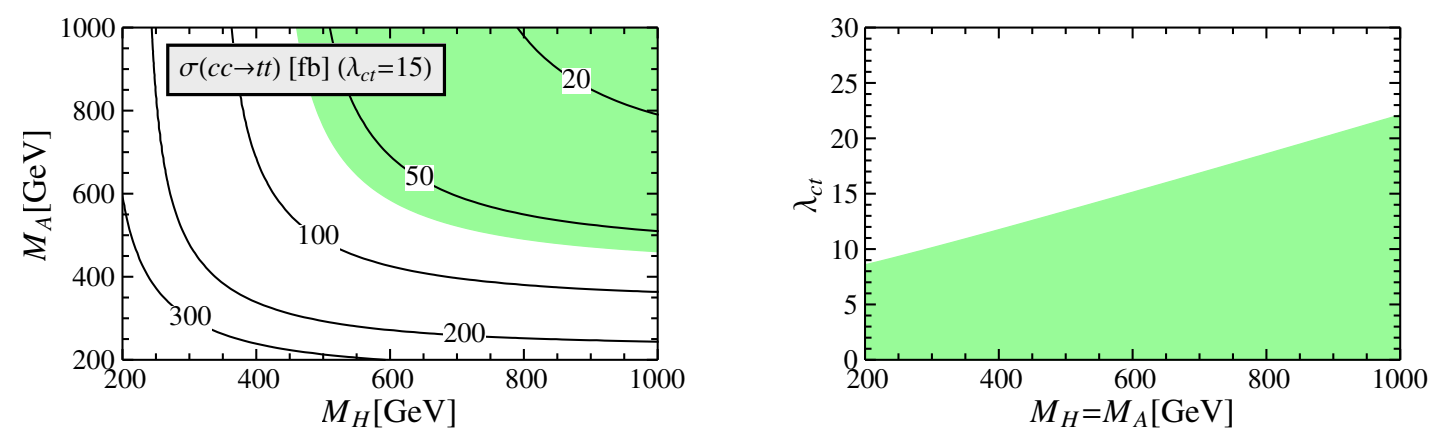

Figure 2. (a) Total cross section for $c c \rightarrow t t$ at the $\mathrm{LHC} 8 \mathrm{TeV}$ run in $\left(M_{H}, M_{A}\right)$ plane. We set $\lambda_{c t}=15$. The shaded region (green) is allowed parameter space at 95\% CL. (b) The allowed parameter space in $\left(M_{H}\left(=M_{A}\right), \lambda_{c t}\right)$ plane in the case where $H$ and $A$ are degenerated in mass.

The experimental searches for the same-sign dileptons and $b$-jets at CMS with $19.5 \mathrm{fb}^{-1}$ [94] and at ATLAS with $20.3 \mathrm{fb}^{-1}$ [56] at $8 \mathrm{TeV}$ can be applied for constraining the same-sign top pair production rate. The non-observation of any significant excess of signal events sets the upper bound of the production cross section. The strongest bound comes from ATLAS result. ATLAS provides different upper bounds depending on the helicity configuration of effective operators within contact interaction model. We conservatively adopt the largest upper bound among the three as follows:

$$
\sigma(p p \rightarrow t t)<62 \mathrm{fb} \quad(\text { ATLAS } 95 \% \text { CL [56]) } .
$$

We re-interpret this result to constrain the cross section $\sigma(c c \rightarrow t t)$ using the formula described above. The constraint is usually strong for small Higgs masses. Since the signal rate is proportional to $\lambda_{c t}^{4}$, the large values of $\lambda_{c t}$ are severely constrained and conversely the small value of $\lambda_{c t}$ is hardly excluded. Figure 2(a) shows the prediction of scattering cross section by setting $\lambda_{c t}=15$ in $\left(M_{H}, M_{A}\right)$ plane and the allowed region with shaded green color. As shown, the interference effect is constructive. For the given $\lambda_{c t}$ value the region $M_{H}, M_{A} \lesssim 400 \mathrm{GeV}$ is excluded. Figure 2 (b) shows the allowed parameter space in $\left(M_{H}, \lambda_{c t}\right)$ plane for the case where $H$ and $A$ are degenerated in mass. Experimental bound provides quite stringent upper limit on $\lambda_{c t}$ as $10 \sim 20$, depending on the heavy Higgs mass.

\section{$5 \quad$ Flavor physics - Tree-level processes}

Since the top-quark FCNC couplings take part in charged Higgs Yukawa sector, they can contribute to the semi-leptonic decay and leptonic decay of $B$ mesons which are tree-level processes. In this section we study the two $\tau$-involved tree-level processes, $B \rightarrow D^{(*)} \tau \nu$ and $B \rightarrow \tau \nu$ to constrain top quark FCNC couplings. The former (latter) is involved with $b \rightarrow c(u)$ charged current. Therefore, any NP model which contains such charged current with a new charged particle can contribute to these processes [95-98].

For those processes with $b \rightarrow c(u)$ charged current, the effective Hamiltonian is described by [23]

$$
\mathcal{H}_{\mathrm{eff}}=C_{\mathrm{VLL}}^{q} \mathcal{O}_{\mathrm{VLL}}^{q}+C_{\mathrm{SRL}}^{q} \mathcal{O}_{\mathrm{SRL}}^{q}+C_{\mathrm{SLL}}^{q} \mathcal{O}_{\mathrm{SLL}}^{q}, \quad(q=u, c)
$$


with the effective four-fermion operators

$$
\begin{aligned}
\mathcal{O}_{\mathrm{VLL}}^{q} & =\left(\bar{q} \gamma_{\mu} P_{L} b\right)\left(\bar{\tau} \gamma^{\mu} P_{L} \nu_{\tau}\right), \\
\mathcal{O}_{\mathrm{SRL}}^{q} & =\left(\bar{q} P_{R} b\right)\left(\bar{\tau} P_{L} \nu_{\tau}\right), \\
\mathcal{O}_{\mathrm{SLL}}^{q} & =\left(\bar{q} P_{L} b\right)\left(\bar{\tau} P_{L} \nu_{\tau}\right) .
\end{aligned}
$$

Within the SM, the vector boson $W^{-}$is exchanged, therefore only $\mathcal{O}_{\mathrm{VLL}}^{q}$ are generated with tree-level Wilson coefficients

$$
C_{\mathrm{VLL}}^{q, \mathrm{SM}}=\frac{4 G_{F} V_{q b}}{\sqrt{2}}
$$

where $G_{F}$ denotes the Fermi coupling constant and $V_{q b}$ are the CKM matrix elements. On the other hand, within the 2HDM type III the scalar charged Higgs boson is exchanged, and therefore $\mathcal{O}_{\text {SLL }}^{q}$ and $\mathcal{O}_{\text {SRL }}^{q}$ are generated. The corresponding tree-level Wilson coefficients are

$$
C_{\mathrm{SLL}}^{c, 2 \mathrm{HDM}}=\frac{V_{t b} \xi_{c t} \xi_{\tau \tau}}{M_{H^{ \pm}}^{2}}, \quad C_{\mathrm{SRL}}^{q, 2 \mathrm{HDM}}=-\frac{V_{q b} \xi_{b b} \xi_{\tau \tau}}{M_{H^{ \pm}}^{2}} .
$$

We neglect $C_{\mathrm{SLL}}^{u, 2 \mathrm{HDM}}$ which is proportional to $\lambda_{u t}$ and extremely suppressed by $u$-quark mass.

For $B \rightarrow D^{(*)} \tau \nu$ decay, we can define a theoretically clean observable by taking the ratio with relatively clean measurement $B \rightarrow D^{(*)} \ell \nu(\ell=e, \mu, \tau)$ to cancel the hadronic uncertainties:

$$
R\left(D^{(*)}\right) \equiv \frac{\mathcal{B}\left(B \rightarrow D^{(*)} \tau \nu\right)}{\mathcal{B}\left(B \rightarrow D^{(*)} \ell \nu\right)}
$$

Note that the CKM matrix element $V_{c b}$ is also canceled out. Then, the theory uncertainty of $R\left(D^{(*)}\right)$ are very small, $6(1) \%$, while the experimental error is quite large, $12(7) \%$ because of missing neutrino in $\tau$ reconstruction.

With the effective Hamiltonian in eq. (5.1), the theoretical prediction of $R\left(D^{(*)}\right)$ relative to the SM value is described as [23, 99-101],

$$
\begin{aligned}
& R(D)=R_{\mathrm{SM}}(D)\left(1+1.5 \operatorname{Re}\left[\frac{C_{\mathrm{SRL}}^{c}+C_{\mathrm{SLL}}^{c}}{C_{\mathrm{VLL}}^{c, \mathrm{SM}}}\right]+1.0\left|\frac{C_{\mathrm{SRL}}^{c}+C_{\mathrm{SLL}}^{c}}{C_{\mathrm{VLL}}^{c, \mathrm{SM}}}\right|^{2}\right), \\
& R\left(D^{*}\right)=R_{\mathrm{SM}}\left(D^{*}\right)\left(1+0.12 \operatorname{Re}\left[\frac{C_{\mathrm{SRL}}^{c}-C_{\mathrm{SLL}}^{c}}{C_{\mathrm{VLL}}^{c, \mathrm{SM}}}\right]+0.05\left|\frac{C_{\mathrm{SRL}}^{c}-C_{\mathrm{SLL}}^{c}}{C_{\mathrm{VLL}}^{c, \mathrm{SM}}}\right|^{2}\right) .
\end{aligned}
$$

Due to the spin of $D^{*}$ meson, the NP effects on $R\left(D^{*}\right)$ are much smaller than the ones on $R(D)$ [100, 102-104]. The relevant Wilson coefficients are given in eqs. (5.3) and (5.4). Since $C_{\mathrm{SRL}}^{c}$ is suppressed by $m_{b} / v$ in Cheng-Sher ansatz and also by CKM matrix element, its contribution is negligibly small.

The BaBar experimental data for $B \rightarrow D^{(*)} \tau \nu$ have shown somewhat large values comparing with the SM expectations for both $R(D)$ and $R\left(D^{*}\right)$ where the combined discrepancy 


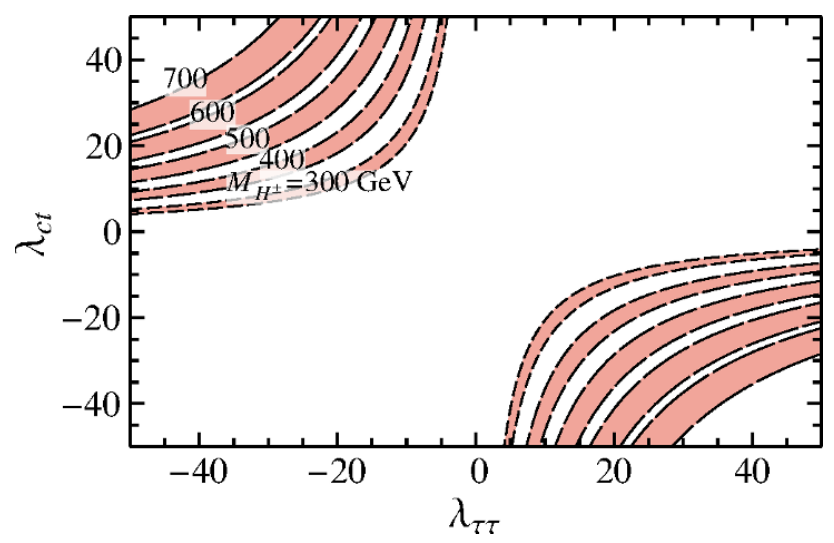

Figure 3. Constraints on $\lambda_{c t}$ and $\lambda_{\tau \tau}$ from $R(D)$ and $R\left(D^{*}\right)$. Pink-colored region is allowed at $95 \%$ CL in $\left(\lambda_{\tau \tau}, \lambda_{c t}\right)$ plane with different charged Higgs masses.

was $3.4 \sigma$ level $[25,105]$. It was also discussed that these can not be simultaneously accommodated by $2 \mathrm{HDM}$ Type II. To explain both discrepancies it was shown that the large top quark FCNC coupling $\lambda_{c t}$ which contributes to $C_{\text {SLL }}^{c}$ in eq. (5.6) is needed [23, 24]. Very recently, the Belle collaboration reported the measurements of both $R(D)$ and $R\left(D^{*}\right)$ [106], and the LHCb collaboration did for $R\left(D^{*}\right)$ [107]. Even though the Belle result is in the middle of the SM expectation and the BaBar result, due to the reduced errors, the average values are still in $3.9 \sigma$ discrepancy [54] (See table 2 for comparison).

The allowed parameter space in $\left(\lambda_{\tau \tau}, \lambda_{c t}\right)$ with different charged Higgs masses constrained by $R\left(D^{(*)}\right)$ is shown in figure 3. For any given charged Higgs mass both $\lambda_{c t}$ and $\lambda_{\tau \tau}$ do not simultaneously become zero. For small $\lambda_{\tau \tau}$ value, $\lambda_{c t}$ must be very large. Interestingly, larger charged Higgs mass requires larger $\lambda_{c t}$. These feature can be understood as a whole since only the product $\lambda_{c t} \lambda_{\tau \tau} / M_{H^{ \pm}}^{2}$ enters the contributions from $2 \mathrm{HDM}$, as show in eq. (5.4). Explicitly, the current $B \rightarrow D^{(*)} \tau \nu$ data put the bound

$$
-0.0030<\lambda_{c t} \lambda_{\tau \tau} / M_{H^{ \pm}}^{2}<-0.0023
$$

which can be seen in figure 3. It is noted that $\lambda_{\tau \tau}$ is associated with the neutral Higgs decay $H / A \rightarrow \tau \tau$. If $\lambda_{\tau \tau}$ is large, the LHC has a good opportunity to detect neutral Higgs bosons in their tauonic decay channels. In the case of small $\lambda_{\tau \tau}$, the coupling $\lambda_{c t}$ should be large, which may be severely constrained by the same sign top pair production as shown in previous section.

Contrary to $B \rightarrow D^{(*)} \tau \nu$ decay, $B \rightarrow \tau \nu$ decay is a helicity suppressed process and more strongly suppressed by CKM factor. Therefore, $B \rightarrow(\mu / e) \nu$ decays are extremely rare, $\mathcal{O}\left(10^{-7}\right)$ and $\mathcal{O}\left(10^{-11}\right)$ respectively, and not yet measured although $B \rightarrow \mu \nu$ will be measured soon at Belle II. Thus, we have no way to cancel the large theory uncertainty of hadronic current of $B \rightarrow \tau \nu$. The uncertainties from the SM prediction and experiment for $\mathcal{B}(B \rightarrow \tau \nu)$ are very large, $24 \%$ and $19 \%$ respectively. Due to these large errors, the constraint from $\mathcal{B}(B \rightarrow \tau \nu)$ is not much significant. 
With the effective hamiltonian in eq. (5.1), the branching ratio of $B \rightarrow \tau \nu$ reads [23]

$$
\mathcal{B}(B \rightarrow \tau \nu)=\frac{G_{F}^{2}\left|V_{u b}\right|^{2}}{8 \pi} m_{\tau}^{2} m_{B} \tau_{B} f_{B}^{2}\left(1-\frac{m_{\tau}^{2}}{m_{B}^{2}}\right)^{2}\left|1+\frac{m_{B}^{2}}{m_{b} m_{\tau}} \frac{C_{\mathrm{SRL}}^{u}-C_{\mathrm{SLL}}^{u}}{C_{\mathrm{VLL}}^{u, \mathrm{SM}}}\right|^{2},
$$

where $f_{B}$ denotes the $B$-meson decay constant. The relevant Wilson coefficients for $2 \mathrm{HDM}$ type III are shown in eqs. (5.3) and (5.4). We note that not only $\xi_{b b}$ but also $\xi_{b s}, \xi_{b d}$ can contribute to $C_{\mathrm{SRL}}^{u}$ within Cheng-Sher ansatz due to the relatively large CKM factors. Even $\xi_{u t}$ can significantly contribute to $C_{\mathrm{SLL}}^{u}$. Due to the combination of these contributions to a single observable $\mathcal{B}(B \rightarrow \tau \nu)$, none of these Yukawa couplings get any meaningful constraints.

\section{$6 \quad$ Flavor physics - Loop-level processes}

\section{1 $\quad B_{d} \rightarrow X_{s} \gamma$}

As for the loop-induced process we first consider $B_{d} \rightarrow X_{s} \gamma$ decay. Taking the normalization with $\mathcal{B}\left(B_{d} \rightarrow X_{c} e \bar{\nu}_{e}\right)$, the dominant theoretical uncertainties from $m_{b}^{5}$ and CKM factor are canceled out. The effective Hamiltonian for the $B_{d} \rightarrow X_{s} \gamma$ decay read $[108,109]$

$$
\mathcal{H}_{\text {eff }}=-\frac{4 G_{F}}{\sqrt{2}} V_{t s}^{*} V_{t b} \sum_{i=1}^{8} C_{i} \mathcal{O}_{i}
$$

where the explicit expressions of the tree or penguin operators $\mathcal{O}_{1-6}$ can be found in ref. [110]. The magnetic penguin operators, $\mathcal{O}_{7}$ and $\mathcal{O}_{8}$, which are characteristic for this decay, are defined as

$$
\mathcal{O}_{7}=\frac{e}{8 \pi^{2}} m_{b} \bar{s}_{\alpha} \sigma^{\mu \nu}\left(1+\gamma_{5}\right) b_{\alpha} F_{\mu \nu}, \quad \mathcal{O}_{8}=\frac{g_{s}}{8 \pi^{2}} m_{b} \bar{s}_{\alpha} \sigma^{\mu \nu}\left(1+\gamma_{5}\right) T_{\alpha \beta}^{a} b_{\beta} G_{\mu \nu}^{a},
$$

where $m_{b}$ denotes the $b$-quark mass in the $\overline{\mathrm{MS}}$ scheme, and $e\left(g_{s}\right)$ is the electromagnetic (strong) coupling constant. The heavy degrees of freedom from the $W^{-}$boson contribution [111-119] and charged Higgs contribution [120-122] are integrated out at $m_{W}$ scale, and we obtain the Wilson coefficients $C_{7,8}\left(\mu=m_{W}\right)$. They evolve into $\mu=m_{b}$ scale by renormalization group equation and consequently resum the large logarithms in perturbative QCD correction to all order [123-125]. The higher order correction at $\mu=m_{b}$ scale should be necessarily done [126-129].

The compilation of all those calculation for $\mathcal{B}\left(B_{d} \rightarrow X_{s} \gamma\right)$ reached at next-to-next-toleading-order (NNLO) in perturbative QCD [130-132]. (For a recent review, we refer to ref. [133].) For given NP contributions to $C_{7,8}^{\mathrm{NP}}$, the theory prediction for $\mathcal{B}\left(B_{d} \rightarrow X_{s} \gamma\right)$ at NNLO is given by [133]

$$
\mathcal{B}\left(B_{d} \rightarrow X_{s} \gamma\right) \times 10^{4}=(3.36 \pm 0.23)-8.22 \operatorname{Re} C_{7}^{\mathrm{NP}}-1.99 \operatorname{Re} C_{8}^{\mathrm{NP}},
$$

where the first number represents the most up-to-date SM prediction. By using current experimental data, we obtain

$$
8.22 \operatorname{Re} C_{7}^{\mathrm{NP}}+1.99 \operatorname{Re} C_{8}^{\mathrm{NP}}=-0.07 \pm 0.32 .
$$

Therefore, it is natural for $C_{7,8}^{2 \mathrm{HDM}}$ to become $\mathcal{O}(0.1)$. 
In the $2 \mathrm{HDM}$ type III, the one-loop contribution to $C_{7,8}$ via charged Higgs exchange is described by [120]

$$
C_{7,8}^{2 \mathrm{HDM}}=\frac{1}{3} A_{u}^{*} F_{7,8}^{(1)}\left(x_{W}\right)-A_{d}^{*} F_{7,8}^{(2)}\left(x_{W}\right)
$$

where the loop functions $F_{7,8}^{(1,2)}$ are given in ref. [120] and $x_{W}=m_{t}^{2} / m_{W}^{2}$. The Yukawa components $A_{u}$ and $A_{d}$ normalized by SM ones are defined as

$$
\begin{aligned}
A_{u} & =\left(\lambda_{t t}+\frac{V_{c s}}{V_{t s}} \sqrt{\frac{m_{c}}{m_{t}}} \lambda_{c t}\right)\left(\lambda_{t t}+\frac{V_{c b}^{*}}{V_{t b}^{*}} \sqrt{\frac{m_{c}}{m_{t}}} \lambda_{c t}\right), \\
A_{d} & =\left(\lambda_{t t}+\frac{V_{c s}}{V_{t s}} \sqrt{\frac{m_{c}}{m_{t}}} \lambda_{c t}\right) \lambda_{b b} .
\end{aligned}
$$

It should be emphasized that the $A_{d}$ term is enhanced by the spin-flip factor $m_{t} / m_{b}$ and becomes comparable to $A_{u}$. Therefore, it is unique for $B_{d} \rightarrow X_{s} \gamma$ that the coupling $\lambda_{b b}$ can be significantly constrained. Another interesting feature is that the coefficient $\lambda_{c t}$ of second factor in $A_{u}$ is highly suppressed while the one in first term contains CKMenhanced factor. The $\lambda_{c t}$ prefers to be $\mathcal{O}(10)$ from $B \rightarrow D^{(*)} \tau \nu$. Thus, the $\lambda_{t t}$ and $\lambda_{b b}$ must be strongly correlated to satisfy eq. (6.4). In order to avoid large cancelation between $1 / 3 \lambda_{t t} F_{7,8}^{(1)}$ and $\lambda_{b b} F_{7,8}^{(2)}$ in eq. (6.5) that causes fine-tuning, we prefer to take the region where $\lambda_{b b}, \lambda_{t t} \sim \mathcal{O}(0.1)$.

To be more specific regarding the fine-tuning argument, we refer to ref. [134] and re-define fine-tuning parameter $\Delta$ for an observable as follows

$$
\Delta=\frac{\max \left(\delta Q_{i}\right)}{Q} .
$$

Here, $Q$ denotes the difference between theory prediction and experimental data and $\delta Q_{i}$ represents each individual contribution of the theory to the $Q$. Therefore, small $\Delta^{-1}$ means significant fine-tuning. (For example, $\Delta=25$ correspond to $4 \%$ fine-tuning.) The allowed parameter space in $\left(\lambda_{t t}, \lambda_{b b}\right)$ plane for given $\lambda_{c t}=10$ and $M_{H^{+}}=400 \mathrm{GeV}$ is shown in figure 4 by requiring $\Delta^{-1}>10 \%$. The gray region causes significant fine-tuning. We note that by avoiding significant fine-tuning, not only $\lambda_{t t}$ is constrained but also $\lambda_{b b}$ is highly restricted as we expected.

\section{$6.2 B_{d, s}-\bar{B}_{d, s}$ mixing}

The $B_{q}-\bar{B}_{q}(q=d, s)$ mixing occurs via box diagrams by exchanging $W$ boson or charged Higgs within $2 \mathrm{HDM}$ between $B_{q}$ and $\bar{B}_{q}$. We note that the tree level diagrams can also contribute through $\bar{b}-s-(H / A)$ vertices within 2 HDM type III. We first study the NP contribution from loop processes while the tree-level contribution is discussed in the next section. The mass difference $\Delta m_{q}$ between the two mass eigenstates $B_{q}^{H}$ and $B_{q}^{L}$ is related with off-diagonal element of mixing matrix $M_{12}^{q}$ such that $\Delta m_{q}=2\left|M_{12}^{q}\right|$. Since the constraints from $B_{d}-\bar{B}_{d}$ mixing appears to be more or less weaker than those from $B_{s}-\bar{B}_{s}$ mixing, we only consider latter one in this work. The effective Hamiltonian with $\Delta B=2$ for the 


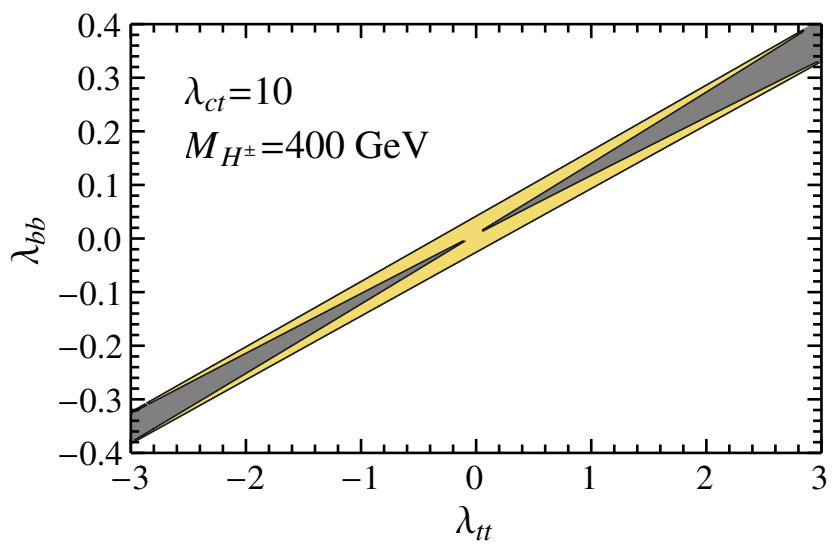

Figure 4. Yellow region is allowed parameter space at 95\% CL from $\mathcal{B}\left(B_{d} \rightarrow X_{s} \gamma\right)$ with fixed $\lambda_{c t}=10$ and $M_{H^{ \pm}}=400 \mathrm{GeV}$ by requiring $\Delta^{-1}>10 \%$. The gray region causes significant fine-tuning.

$B_{s}-\bar{B}_{s}$ mixing is described by $[135]$

$$
\mathcal{H}^{\Delta B=2}=\frac{G_{F}^{2}}{16 \pi^{2}} m_{W}^{2}\left(V_{t b}^{*} V_{t s}\right)^{2} \sum_{i} C_{i} \mathcal{O}_{i}+\text { h.c.. }
$$

In the SM, only $\mathcal{O}_{1}^{\mathrm{VLL}}$ operator can contribute, where

$$
\mathcal{O}_{1}^{\mathrm{VLL}}=\left(\bar{b}^{\alpha} \gamma_{\mu} P_{L} s^{\alpha}\right)\left(\bar{b}^{\beta} \gamma^{\mu} P_{L} s^{\beta}\right) .
$$

The corresponding Wilson coefficient is $C_{1}^{\mathrm{VLL}}\left(m_{W}\right)=4 S_{0}\left(x_{W}\right)$ where $x_{W}=m_{t}^{2} / m_{W}^{2}$. The function $S_{0}(x)$ can be found in ref. [108]. Then the $\Delta m_{s}$ is obtained as

$$
\Delta m_{s}=2\left|\left\langle B_{s}\left|\mathcal{H}^{\Delta B=2}\right| \bar{B}_{s}\right\rangle\right|=\frac{G_{F}^{2}}{6 \pi^{2}}\left|V_{t b}^{*} V_{t s}\right|^{2} f_{B_{s}}^{2} \hat{B}_{B_{s}} m_{B_{s}} \eta_{b} m_{W}^{2} S_{0}\left(x_{W}\right) .
$$

Here, $\eta_{b}=0.552$ is a short-distance QCD contribution. As for the long distance nonperturbative quantity $f_{B_{s}} \hat{B}_{B_{s}}^{1 / 2}$, we use Lattice QCD result.

Within the $2 \mathrm{HDM}$, two additional operators are generated by the box diagrams with charged Higgs boson exchanged:

$$
\mathcal{O}_{1}^{\mathrm{SRR}}=\left(\bar{b}^{\alpha} P_{R} s^{\alpha}\right)\left(\bar{b}^{\beta} P_{R} s^{\beta}\right), \quad \mathcal{O}_{2}^{\mathrm{SRR}}=\left(\bar{b}^{\alpha} \sigma_{\mu \nu} P_{R} s^{\alpha}\right)\left(\bar{b}^{\beta} \sigma^{\mu \nu} P_{R} s^{\beta}\right) .
$$

Using the formulae in ref. [136], the corresponding Wilson coefficients are obtained as

$$
\begin{gathered}
C_{1, H H}^{\mathrm{VLL}}=A_{u}^{2} x_{W} x_{H^{ \pm}}\left[\frac{x_{H^{ \pm}}+1}{\left(x_{H^{ \pm}}-1\right)^{2}}-\frac{2 x_{H^{ \pm}} \log x_{H^{ \pm}}}{\left(x_{H^{ \pm}}-1\right)^{3}}\right] \\
C_{1, W H}^{\mathrm{VLL}}=2 A_{u} x_{W} x_{H^{ \pm}}\left[\frac{-4+x_{W}}{\left(x_{H^{ \pm}}-1\right)\left(x_{W}-1\right)}+\frac{\left(x_{W}-4 x_{H^{ \pm}}\right) \log x_{H^{ \pm}}}{\left(x_{H^{ \pm}}-1\right)^{2}\left(x_{H^{ \pm}}-x_{W}\right)}\right. \\
\left.+\frac{3 x_{W} \log x_{W}}{\left(x_{W}-1\right)^{2}\left(x_{H^{ \pm}}-x_{W}\right)}\right] \\
C_{1, H H}^{\mathrm{SRR}}=4 A_{d^{2} x_{H^{ \pm}}^{2}}\left(\frac{m_{b}^{2}}{m_{W}^{2}}\right)\left[\frac{2}{\left(x_{H^{ \pm}}-1\right)^{2}}-\frac{\left(x_{H^{ \pm}}+1\right) \log x_{H^{ \pm}}}{\left(x_{H^{ \pm}}-1\right)^{3}}\right]
\end{gathered}
$$



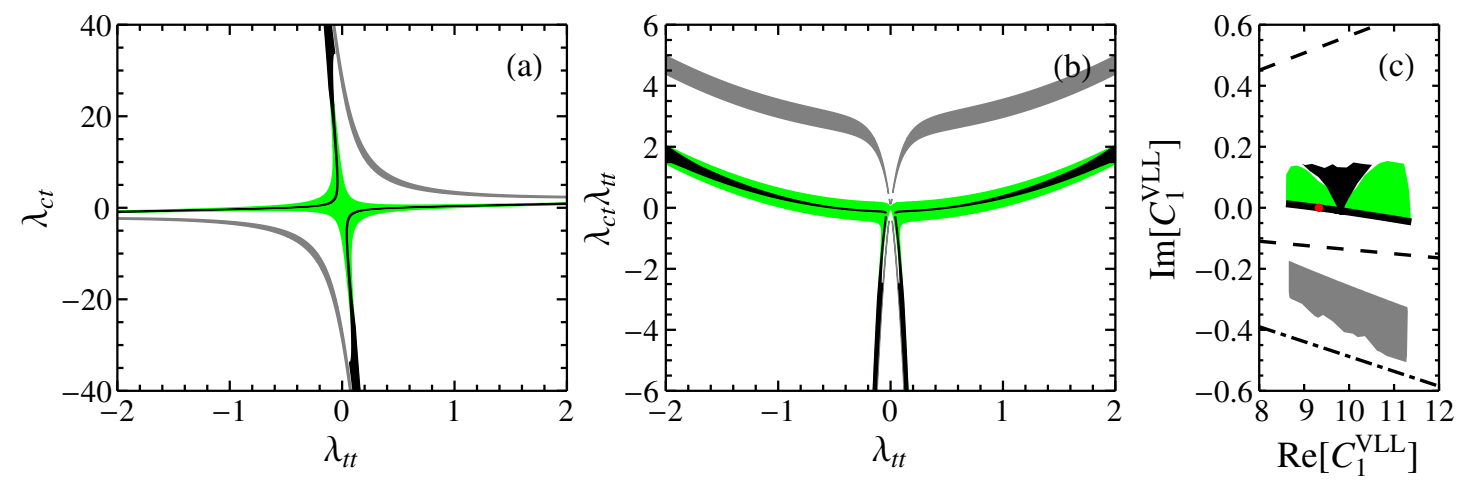

Figure 5. Allowed parameter space at $95 \% \mathrm{CL}$ by $\Delta m_{s}$ experimental data for fixed $M_{H^{ \pm}}=$ $500 \mathrm{GeV}$ in (a) $\left(\lambda_{t t}, \lambda_{c t}\right)$, (b) $\left(\lambda_{t t}, \lambda_{c t} \lambda_{t t}\right)$ and (c) $\left(\operatorname{Re}\left[C_{1}^{\mathrm{VLL}}\right], \operatorname{Im}\left[C_{1}^{\mathrm{VLL}}\right]\right)$ planes. Green color (S1) corresponds to the solution without significant fine-tuning. Black color (S2) and gray color (S3) represent the parameter space with significant fine-tuning, $\Delta^{-1}<10 \%$, where S3 causes large $\operatorname{Im} M_{12}^{s}$ while S2 does not. The dashed (dot dashed) line denotes $68 \%$ CL $(95 \% \mathrm{CL})$ bound from $\phi_{s}^{c \bar{s} s}$. The red point represents the SM prediction

where $x_{H^{ \pm}}=m_{t}^{2} / M_{H^{ \pm}}^{2}$. The subscript $W H$ or $H H$ represent the exchanged particles in the box diagram. We note that $C_{2}^{\mathrm{SRR}}=0$ at the matching scale $\mu_{W}$. Contrary to the $B_{d} \rightarrow X_{s} \gamma$, the $A_{d}$ contribution in $C_{1}^{\mathrm{SRR}}$ has significant suppression factor $m_{b}^{2} / m_{W}^{2}$, thus its contribution is negligible. Although the operators $\mathcal{O}_{1}^{\mathrm{SRR}}$ and $\mathcal{O}_{2}^{\mathrm{SRR}}$ are generated through operator mixing during renormalization group evolution as described in detail in refs. $[135,137-141]$ at NLO QCD, the effects are minor and we do not include them.

Therefore, only $A_{u}$ is numerically relevant in $B_{s}-\bar{B}_{s}$ mixing. It contains $\lambda_{t t}$ and $\lambda_{c t}$ as defined in eq. (6.6) which are constrained by experimental data of $\Delta m_{s}$ given in table 2. The allowed region for the parameter space in $\left(\lambda_{t t}, \lambda_{c t}\right)$ plane as well as $\left(\lambda_{t t}, \lambda_{c t} \lambda_{t t}\right)$ plane are shown in figure $5(\mathrm{a})$ and (b). We perform more detailed study on the allowed parameter space by considering the fine-tuning argument to fit the data. As shown in eq. (6.6), there are two solutions for $A_{u}=0$ which give the result consistent with experimental data:

$$
\begin{aligned}
\lambda_{t t} & \simeq-\frac{V_{c s}}{V_{t s}} \sqrt{\frac{m_{c}}{m_{t}}} \lambda_{c t} \simeq(2.14-0.04 i) \lambda_{c t}, \\
\text { or } \quad \lambda_{t t} & \simeq-\frac{V_{c b}^{*}}{V_{t b}^{*}} \sqrt{\frac{m_{c}}{m_{t}}} \lambda_{c t} \simeq-0.004 \lambda_{c t} .
\end{aligned}
$$

The parameter space near these two solutions are allowed, but can cause significant finetuning. We represent the allowed parameter without significant fine-tuning, or $\Delta^{-1}>10 \%$ by green color, and for $\Delta^{-1}<10 \%$ by black color.

In the region where the signs of $\lambda_{c t}$ and $\lambda_{t t}$ are same, the two 2HDM contributions $C_{1, W H}^{\mathrm{VLL}}$ and $C_{1, H H}^{\mathrm{VLL}}$ are destructive with each other. The parameter space that brings the cancelation between the two can be another solution to fit the data, but also causes significant fine-tuning. We represent the parameter space near the solution with significant fine-tuning, $\Delta^{-1}<10 \%$, with gray color. For this solution space, the real parts of the two 2HDM contributions are strongly canceled, but sizable imaginary parts still remain as can be seen in the figure $5(\mathrm{c})$. This sizable imaginary part can cause large time-dependent 
$C P$-asymmetry phase $\phi_{s}^{c \bar{c} s}$ in $b \rightarrow c$ decays from the relation $\phi_{s}^{c \bar{c} s} \equiv \arg \left(M_{12}^{s}\right)$. We show the bounds at $68 \%$ and $95 \%$ CL in figure 5(c) with current average value [53]

$$
\phi_{s}^{c \bar{c} s}=-0.015 \pm 0.035 .
$$

As shown, the gray region is excluded by $\phi_{s}^{c \bar{c} s}$ at $68 \% \mathrm{CL}$, but survives at $95 \% \mathrm{CL}$. This region will be more significantly covered by future experimental data.

For later convenience, we summarize the features of each parameter regions and their color notation with the definition of S1, S2 and S3 as follows

$$
\begin{aligned}
& \mathrm{S} 1: \text { (green color) } \Delta^{-1}>10 \%, \\
& \mathrm{~S} 2:\left(\text { black color) } \Delta^{-1}<10 \%, A_{u} \simeq 0,\right. \\
& \mathrm{S} 3:\left(\text { gray color) } \Delta^{-1}<10 \%, \operatorname{Re} C_{1, W H}^{\mathrm{VLL}}+\operatorname{Re} C_{1, H H}^{\mathrm{VLL}} \simeq 0, \text { large } \operatorname{Im} M_{12}^{s} .\right.
\end{aligned}
$$

\section{Combined analysis and future prospect}

We first combine the constraints from $B_{d} \rightarrow X_{s} \gamma, B_{s}-\bar{B}_{s}$ mixing, and $c c \rightarrow t t$ on the couplings $\lambda_{c t}$ and $\lambda_{t t}$. We also include the constraints from EW precision measurements, $Z \rightarrow b \bar{b}$ and $\Delta \rho$. We refer to ref. [43] for the details of these EW precision measurements. We scan the parameter space as described in eq. (3.1). The allowed parameter space is obtained by requiring that it accommodates all the experimental data with 95\% CL. The result is shown in figure 6 (a) for $M_{H^{ \pm}}=500 \mathrm{GeV}$. As discussed in previous section we divide allowed parameter region into S1, S2 and S3 whose features are portrayed in eq. (6.15).

For the region $\mathrm{S} 1$, the requirement $\Delta^{-1}>10 \%$ in $B_{s}-\bar{B}_{s}$ mixing gives the upper bound on $\lambda_{c t}$ and is slightly stronger than the one from $\sigma(c c \rightarrow t t)$ combined with $\Delta \rho$. The upper bound on $\lambda_{t t}$ for the region $\mathrm{S} 1$ is given by $R_{b}$. On the other hand, for the regions $\mathrm{S} 2$ and $\mathrm{S} 3$, the couplings $\lambda_{c t}$ and $\lambda_{t t}$ are bounded by $\sigma(c c \rightarrow t t)$ accompanied with $\Delta \rho$ and $R_{b}$. Therefore, the same sign top pair production plays crucial role to constrain $\lambda_{c t}$ regardless of fine-tuning. But if we avoid significant fine-tuning (for S1), $B_{s}-\bar{B}_{s}$ mixing put the significant bound. The projection for the exclusion limit at $14 \mathrm{TeV}$ with $300 \mathrm{fb}^{-1}$ is estimated by assuming that the statistical error is dominant (See ref. $[142,143]$ for more details about the projection method). The result is outstanding. The upper bound of $\lambda_{c t}$ reach $8 \sim 15$ with $300 \mathrm{fb}^{-1}$ at $14 \mathrm{TeV}$ as shown in figure 6 . We note that $B_{d} \rightarrow X_{s} \gamma$ does not put bound on $\lambda_{c t}$ nor $\lambda_{t t}$ for any parameter sets due to sizable contributions from $\lambda_{b b}$ term.

We turn to the $B \rightarrow D^{(*)} \tau \nu$ decays. With fixed $\lambda_{\tau \tau}, B \rightarrow D^{(*)} \tau \nu$ decays also put bounds on $M_{H^{ \pm}}$and $\lambda_{c t}$. By taking $\lambda_{\tau \tau}=40$, the allowed parameter space is shown in blue-colored region in figure 6 (with $M_{H^{ \pm}}=500 \mathrm{GeV}$ ). As shown in figure 6(b), $\left|\lambda_{c t}\right|$ has different upper limits for each parameter set depending on $M_{H^{ \pm}}$. They lead to lower limits on $\left|\lambda_{\tau \tau}\right|$ as can be seen in eq. (5.7) and figure 3. The allowed parameter spaces in $\left(M_{H^{ \pm}},\left|\lambda_{\tau \tau}\right|\right)$ plane are presented in figure 7 . For fixed $M_{H^{ \pm}}$, the lower bounds for $\mathrm{S} 2$ and S3 are same and slightly different from S1. It should be noted that these lower bounds become stronger as $M_{H^{ \pm}}$increases. Conversely, the $M_{H^{ \pm}}$is upper bounded when $\lambda_{\tau \tau}$ is fixed. In the case of relatively heavy charged Higgs, the lower bound on $\lambda_{\tau \tau}$ is very strong. 

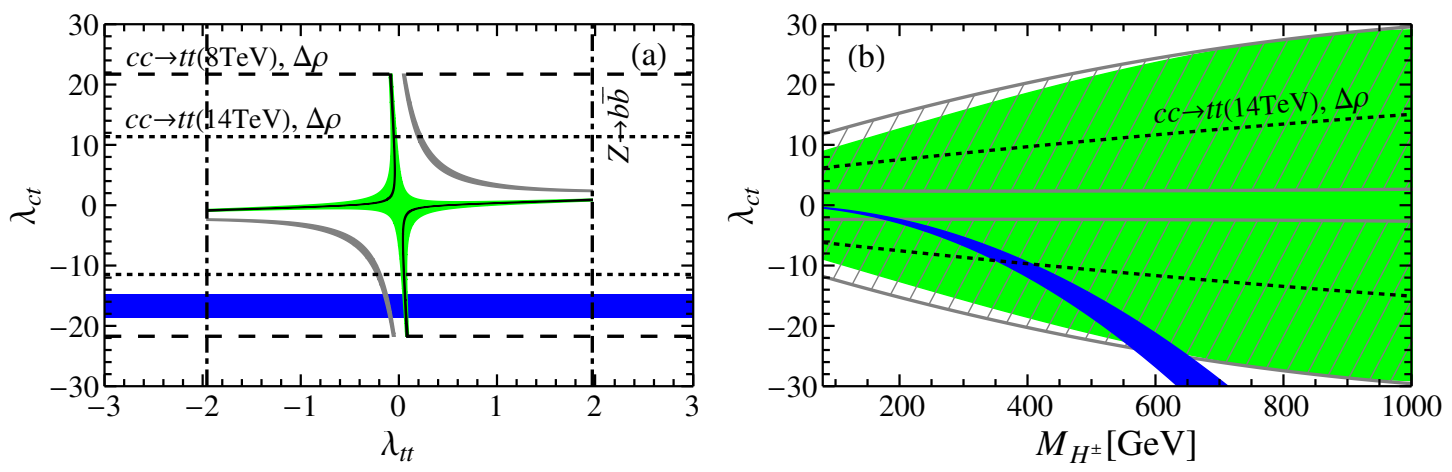

Figure 6. Combined constraints from $B_{s}-\bar{B}_{s}$ mixing, $c c \rightarrow t t, Z \rightarrow b \bar{b}$ and the oblique parameter $\Delta \rho$ on the $2 \mathrm{HDM}$ parameters. The allowed regions are divided into three parts and shown in the green (S1), black (S2) and gray regions (S3). (a) Allowed parameter space in $\left(\lambda_{t t}, \lambda_{c t}\right)$ plane for the fixed $M_{H^{ \pm}}=500 \mathrm{GeV}$. The constraints from $c c \rightarrow t t, \Delta \rho$ and $Z \rightarrow b \bar{b}$ are shown in dashed and dot-dashed lines respectively. The projection for $c c \rightarrow t t, \Delta \rho$ at $14 \mathrm{TeV}$ with $300 \mathrm{fb}^{-1}$ data is shown by a dotted line. The allowed parameter space by $B \rightarrow D^{(*)} \tau \nu$ (with $\lambda_{\tau \tau}=40$ ) are indicated by the blue region. (b) Allowed parameter space in $\left(M_{H^{ \pm}}, \lambda_{c t}\right)$ plane. Note that the upper and lower bounds of black region are same with gray region so they are not shown in the plot.

With the constrains of $c c \rightarrow t t$ at $14 \mathrm{TeV}$ with $300 \mathrm{fb}^{-1}$ data, the lower bound on $\lambda_{\tau \tau}$ would become twice of current bound as shown in figure 7 . For $M_{H^{ \pm}}>500 \mathrm{GeV}$, the coupling $\lambda_{\tau \tau}$ should be greater than 30 , which can significantly enhance $H / A \rightarrow \tau \tau$ decays. Therefore, this can be constrained by heavy Higgs search with $\tau \tau$ final states at the LHC. However the signal strength of $g g \rightarrow H / A \rightarrow \tau \tau$ process strongly depends on heavy Higgses masses and is effectively proportional to $\lambda_{t t}^{2}$. Since there are much parameter space near $\lambda_{t t} \sim 0$ in the set S1 (green region) as shown in figure 6 that may avoid the constrains from $g g \rightarrow H / A \rightarrow \tau \tau$, the constraints would be restricted. Perhaps, some part of parameter space, especially small $\lambda_{c t}$ and large $\lambda_{t t}, \lambda_{\tau \tau}$ region will be excluded. On top of that, for such very large $\tau$ Yukawa coupling, the perturbativity would be threatened.

We now discuss about the constraints from $t \rightarrow c g$. With the above allowed regions S1, $\mathrm{S} 2$ and $\mathrm{S} 3$, we make theoretical predictions for $\mathcal{B}(t \rightarrow c g)$. Since the combined constraints put upper bounds on both $\lambda_{c t}$ and $\lambda_{t t}$, Therefore, $\lambda_{c t} \lambda_{t t}$ is upper bounded in all three parameter sets S1, S2 and S3. Note that the set S3 represents also the lower bounds for both $\lambda_{c t}$ and $\lambda_{t t}$ that comes from $Z \rightarrow b \bar{b}$ and $c c \rightarrow t t$ as shown figure 6(a). The upper bound of $\mathcal{B}(t \rightarrow c g)$ for S1, S2 and the allowed region for S3 are presented as a function of $M_{H^{ \pm}}$in figure 8 .

The current LHC upper limit is much larger than these theory predictions. Thus, it does not give any constraints. The projection for the upper limit at $14 \mathrm{TeV}$ with $300 \mathrm{fb}^{-1}$ data is also drawn in figure 8 in dotted line. As shown, it would be hopeless to see or constrain the top quark FCNC couplings from the $t \rightarrow c g$ measurement.

So far, we have neglected the tree-level contribution to $B_{s}-\bar{B}_{s}$ mixing through the down-type FCNC couplings $\bar{b}-s-(H / A)$ with the Yukawa coupling $\xi_{b s}$. Even though $\xi_{b s}$ is severely suppressed in Cheng-Sher ansatz such as $\xi_{b s} / \lambda_{b s}=3.6 \times 10^{-3}$, the tree level 


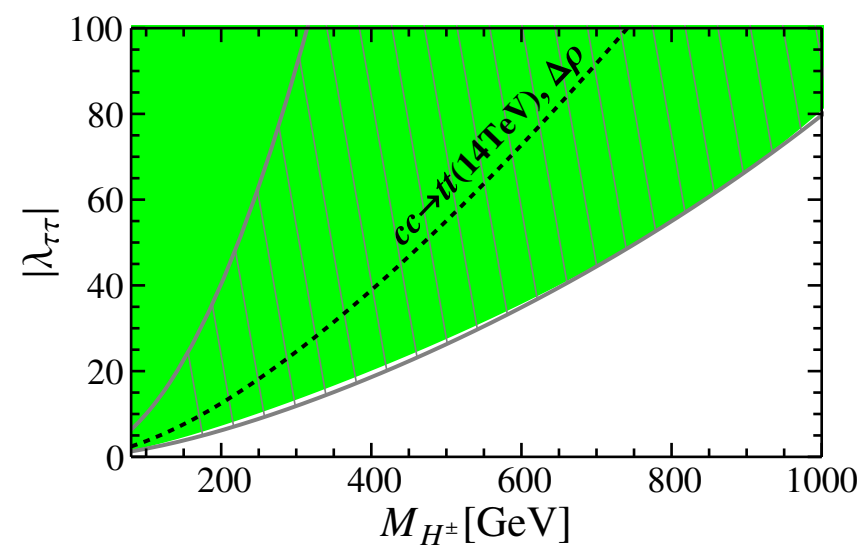

Figure 7. Allowed parameter space $\left(M_{H^{ \pm}},\left|\lambda_{\tau \tau}\right|\right)$ by the combined constraints from loop-induced processes and $B \rightarrow D^{(*)} \tau \nu$ decays. See eq. (6.15) for the definition of each parameter set. The projection for $c c \rightarrow t t, \Delta \rho$ at $14 \mathrm{TeV}$ with $300 \mathrm{fb}^{-1}$ data is shown by a dotted line. The lower bound of black region is same with gray region so is not shown in the plot.

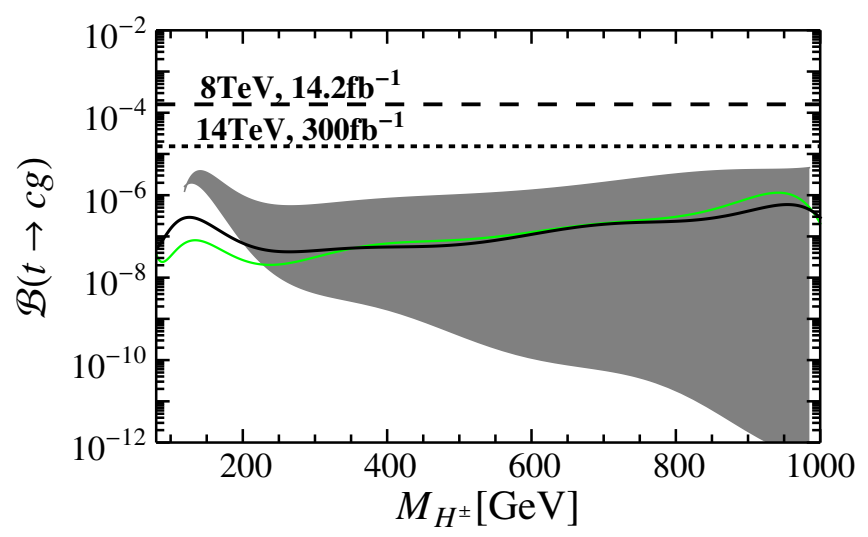

Figure 8. $2 \mathrm{HDM}$ prediction on $\mathcal{B}(t \rightarrow c g)$ as a function of $M_{H^{ \pm}}$. The green and black lines denote the upper bounds from the solutions of the combined constraints S1 and S2, respectively. The gray region corresponds to the solution S3. The dashed line denotes the current upper bound at LHC, while the dotted line is for the future sensitivity at $14 \mathrm{TeV}$ with $300 \mathrm{fb}^{-1}$ data.

contribution with $\mathcal{O}(1) \lambda_{s b}$ has no CKM suppression, and is comparable to the loop contribution. By including the tree level contribution, the allowed parameter space in $\left(\lambda_{t t}, \lambda_{c t}\right)$ plane is significantly extended since the large NP contribution from the loop processes can be canceled by the tree level contribution. Therefore, including the tree level contribution in $B_{s}-\bar{B}_{s}$ mixing always weakens the constraints on $\lambda_{t t}, \lambda_{c t}$. To understand the effect of $\lambda_{s b}$ quantitatively, we show a plot in figure 9 for allowed region of $\lambda_{c t} \lambda_{t t}$ with respect to the fixed $\lambda_{s b}$ value by imposing the constraints from $c c \rightarrow t t, Z \rightarrow b \bar{b}$ and $\Delta \rho$. We see that for $\lambda_{s b}>0.003 M_{H, A}$ large $\lambda_{c t} \lambda_{t t}$ is required to cancel the large tree-level contribution. In fact, for $\lambda_{s b} \simeq 0.003 M_{H, A}$, the magnitude of tree-level contributions is already comparable to the magnitude of the SM contributions. For $\lambda_{s b}<0.003 M_{H, A}$, the bound on $\lambda_{c t} \lambda_{t t}$ is not much changed from the one given in previous section. 


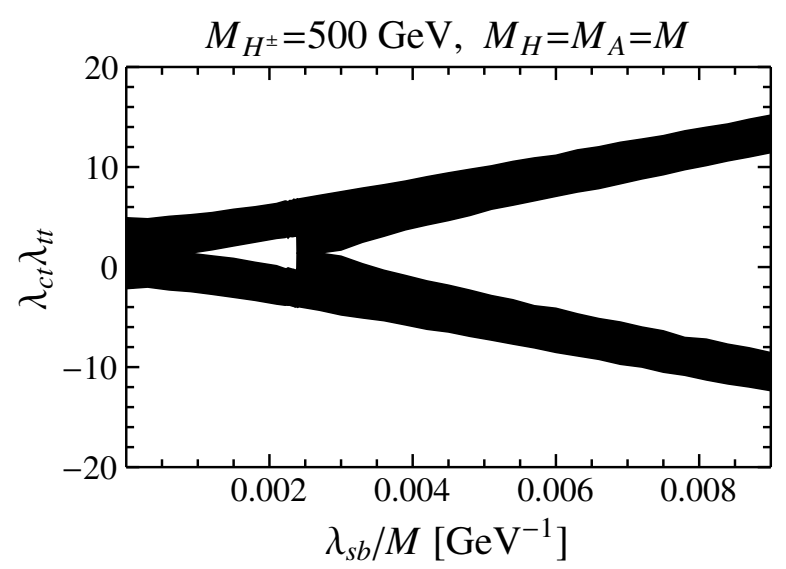

Figure 9. Allowed parameter space in $\left(\lambda_{s b} / M, \lambda_{c t} \lambda_{t t}\right)$ by the combined constraints from $B_{s}-\bar{B}_{s}$ mixing, $c c \rightarrow t t, Z \rightarrow b \bar{b}$ and the oblique parameter $\Delta \rho . M_{H}=M_{A}=M$ and $M_{H^{ \pm}}=500 \mathrm{GeV}$ are taken. For $B_{s}-\bar{B}_{s}$ mixing, both the tree-level and loop-level contributions are included.

\section{Conclusion}

The general $2 \mathrm{HDM}$ as an extension to the SM is a potential NP candidate. To avoid severe constraints from down-type quark FCNC, we adopt Cheng-Sher ansatz. This NP scenario permits presumably large top quark FCNC coupling $\lambda_{c t}$, which is the main target to be explored in this work with collider phenomenology as well as flavor constraints and EW precision measurements. To this end, we consider anomalous single top production which can limit $\mathcal{B}(t \rightarrow c g)$ and the same sign top pair production via $c c \rightarrow t t$ at the LHC in association with not only flavor tree-level processes, $B \rightarrow D^{(*)} \tau \nu, B \rightarrow \tau \nu$ but also flavor loop-level processes, $B_{d} \rightarrow X_{s} \gamma, B_{s}-\bar{B}_{s}$ mixing.

We find that among them the $B \rightarrow D^{(*)} \tau \nu, B_{s}-\bar{B}_{s}$ mixing and $c c \rightarrow t t$ play important role to constrain $\lambda_{c t}$. Especially, still large value of $\lambda_{c t}$ is preferred by average value of $R\left(D^{(*)}\right)$ measurement with the new data for $B \rightarrow D^{(*)} \tau \nu$ from Belle and LHCb. To bring solid understanding of the result, we separate the allowed parameter space into three sets, S1, S2 and S3, regarding the fine-tuning to fit the data and the features reflected in the observables of $B_{s}-\bar{B}_{s}$ mixing. S1 does not suffer from the fine-tuning while S2 and S3 cause significant fine-tuning to fit the data. More specifically, S3 shows large imaginary part of $M_{12}^{s}$ while S1 and S2 do not.

For the allowed parameter sets $\mathrm{S} 1, \mathrm{~S} 2$ and $\mathrm{S} 3, \lambda_{c t}$ is severely upper-bounded by either $c c \rightarrow t t$ or $B_{s}-\bar{B}_{s}$ mixing. Therefore, to fit the $R\left(D^{(*)}\right)$ values, the Yukawa coupling $\lambda_{\tau \tau}$ is lower bounded for given charged Higgs mass $M_{H^{ \pm}}$and conversely $M_{H^{ \pm}}$is upper bounded for fixed $\lambda_{\tau \tau}$. The large $\lambda_{\tau \tau}$ will be constrained by $g g \rightarrow H / A \rightarrow \tau \tau$, however it strongly depends on neutral Higgses masses and $\lambda_{t t}$. The extended study with heavy Higgs search data at the LHC can be a future work. Since $\lambda_{c t} \lambda_{t t}$ is small for all the parameter sets and the theory prediction is loop-suppressed, the upper limits for $\mathcal{B}(t \rightarrow c g)$ do not provide constraints on the remaining parameter space with current experimental data nor in future LHC experiment. On the other hand, large $\lambda_{c t}$ is mostly constrained by $c c \rightarrow t t$ process regardless of fine-tuning. $c c \rightarrow t t$ would play more important role to probe top quark FCNC at the LHC $14 \mathrm{TeV}$ Run. 


\section{Acknowledgments}

CSK and XY are supported by the NRF grant funded by the Korean government of the MEST (No. 2011-0017430) and (No. 2011-0020333). YWY is supported in part by NRF2013R1A1A2061331 and in part by NRF-2012R1A2A1A01006053. YWY thank Sunghoon Jung for useful discussions. We thank KIAS Center for Advanced Computation for providing computing resources.

\section{A Form factors in $t \rightarrow c g$}

In general 2HDM, the form factors for $t c g$ vertex was first calculated in refs. [27, 144]. Here, we recalculate these form factors and write them in terms of scalar one-loop functions. Each form factor in eq. (4.1) is summation of four different contributions from the penguin diagrams with $A, H$ and $H^{ \pm}$exchanges, e.g. $\mathcal{A}=\mathcal{A}_{A}+\mathcal{A}_{H}+\mathcal{A}_{H^{ \pm}}$. They are calculated as

$$
\begin{array}{rlrl}
\mathcal{A}_{A} & =-g_{s} \xi_{A}^{A} f_{1}^{A}, & \mathcal{A}_{H}=g_{s} \xi_{H}^{V} f_{1}^{H}, & \mathcal{A}_{H^{ \pm}}=g_{s}\left|V_{t b}\right|^{2} \xi_{H^{ \pm}} f_{1}^{H^{ \pm}}, \\
\mathcal{B}_{A}=g_{s} \xi_{A}^{V} f_{1}^{A}, & \mathcal{B}_{H}=-g_{s} \xi_{H}^{A} f_{1}^{H}, & \mathcal{B}_{H^{ \pm}}=g_{s}\left|V_{t b}\right|^{2} \xi_{H^{ \pm}} f_{1}^{H^{ \pm}}, \\
\mathcal{C}_{A}=-g_{s} \xi_{A}^{A} f_{2}^{A}, & \mathcal{C}_{H}=g_{s} \xi_{H}^{V} f_{2}^{H}, & \mathcal{C}_{H^{ \pm}}=g_{s}\left|V_{t b}\right|^{2} \xi_{H^{ \pm}} f_{2}^{H^{ \pm}}, \\
\mathcal{D}_{A}=-g_{s} \xi_{A}^{V} f_{2}^{A}, & \mathcal{D}_{H}=g_{s} \xi_{H}^{A} f_{2}^{H}, & \mathcal{D}_{H^{ \pm}}=-g_{s}\left|V_{t b}\right|^{2} \xi_{H^{ \pm}} f_{2}^{H^{ \pm}}
\end{array}
$$

To compare with refs. [27, 144], we neglect the small term $V_{c b} \xi_{c t}$ in $\bar{t} b H^{+}$vertex of eq. (2.3) and show the result in general with complex Yukawa couplings

$$
\begin{aligned}
& \xi_{H}^{V}=\frac{1}{4} \xi_{t t}\left(\xi_{c t}+\xi_{t c}^{*}\right), \quad \xi_{A}^{A}=\frac{1}{4} \xi_{t t}\left(\xi_{c t}-\xi_{t c}^{*}\right), \quad \xi_{H^{ \pm}}=\frac{1}{4} \xi_{c t} \xi_{t t}, \\
& \xi_{H}^{A}=\frac{1}{4} \xi_{t t}\left(\xi_{c t}-\xi_{t c}^{*}\right), \quad \xi_{A}^{V}=\frac{1}{4} \xi_{t t}\left(\xi_{c t}+\xi_{t c}^{*}\right) .
\end{aligned}
$$

The loop functions are defined as

$$
\begin{aligned}
f_{1}^{A} & =q^{2}\left(C_{0}^{A}-2 C_{11}^{A}-C_{12}^{A}+C_{2}^{A}\right), & f_{2}^{A} & =m_{t}^{2}\left(C_{0}^{A}-C_{12}^{A}+C_{2}^{A}\right), \\
f_{1}^{H} & =q^{2}\left(C_{0}^{H}+2 C_{11}^{H}+C_{12}^{H}+C_{2}^{H}+4 C_{1}^{H}\right), & f_{2}^{H} & =m_{t}^{2}\left(C_{0}^{H}+C_{12}^{H}+C_{2}^{H}\right), \\
f_{1}^{H^{ \pm}} & =q^{2}\left(4 C_{1}^{H^{ \pm}}+4 C_{11}^{H^{ \pm}}+2 C_{12}^{H^{ \pm}}\right), & f_{2}^{H^{ \pm}} & =m_{t}^{2}\left(2 C_{12}^{H^{ \pm}}\right) .
\end{aligned}
$$

The scalar one-loop functions are abbreviated as

$$
C_{i j}^{H, A}=C_{i j}\left(q^{2}, m_{t}^{2}, 0, m_{t}^{2}, m_{t}^{2}, m_{H, A}^{2}\right), \quad C_{i j}^{H^{ \pm}}=C_{i j}\left(q^{2}, m_{t}^{2}, 0,0,0, M_{H^{ \pm}}^{2}\right),
$$

which are defined in refs. [145-147] and can be numerically evaluated by the LoopTools package [147]. In the penguin diagrams with charged Higgs $H^{ \pm}$, we omit the terms proportional to $\xi_{b b}$ as in refs. $[27,144]$, since these terms are suppressed by $m_{b} / v$. In addition, we have analytically checked that the form factors presented in this paper are in agreement with those obtained in ref. [27] except one minor discrepancy: for the parameter $\beta^{H, A}$ defined in ref. [27], we obtained $\beta^{H, A}=x^{2} m_{t}^{2}+(1-x) M_{H, A}^{2}$. But this does not come into play in our numerical analysis. 
Open Access. This article is distributed under the terms of the Creative Commons Attribution License (CC-BY 4.0), which permits any use, distribution and reproduction in any medium, provided the original author(s) and source are credited.

\section{References}

[1] GFitTer Group collaboration, M. Baak et al., The global electroweak fit at NNLO and prospects for the LHC and ILC, Eur. Phys. J. C 74 (2014) 3046 [arXiv:1407.3792] [INSPIRE].

[2] ATLAS collaboration, Observation of a new particle in the search for the standard model Higgs boson with the ATLAS detector at the LHC, Phys. Lett. B 716 (2012) 1 [arXiv:1207.7214] [INSPIRE].

[3] CMS collaboration, Observation of a new boson at a mass of $125 \mathrm{GeV}$ with the CMS experiment at the LHC, Phys. Lett. B 716 (2012) 30 [arXiv:1207.7235] [INSPIRE].

[4] CMS collaboration, Precise determination of the mass of the Higgs boson and tests of compatibility of its couplings with the standard model predictions using proton collisions at 7 and $8 \mathrm{TeV}$, Eur. Phys. J. C 75 (2015) 212 [arXiv:1412.8662] [INSPIRE].

[5] ATLAS collaboration, Measurements of the Higgs boson production and decay rates and coupling strengths using pp collision data at $\sqrt{s}=7$ and $8 \mathrm{TeV}$ in the ATLAS experiment, ATLAS-CONF-2015-007 (2015).

[6] S.P. Martin, A Supersymmetr y primer, Adv. Ser. Direct. High Energy Phys. 21 (2010) 1 [Adv. Ser. Direct. High Energy Phys. 18 (1998) 1] hep-ph/9709356 [INSPIRE].

[7] G.C. Branco et al., Theory and phenomenology of two-Higgs-doublet models, Phys. Rept. 516 (2012) 1 [arXiv: 1106.0034] [InSPIRE].

[8] S.L. Glashow, J. Iliopoulos and L. Maiani, Weak interactions with lepton-hadron symmetry, Phys. Rev. D 2 (1970) 1285 [inSPIRE].

[9] G. Eilam, J.L. Hewett and A. Soni, Rare decays of the top quark in the standard and two Higgs doublet models, Phys. Rev. D 44 (1991) 1473 [Erratum ibid. D 59 (1999) 039901] [INSPIRE].

[10] S.L. Glashow and S. Weinberg, Natural conservation laws for neutral currents, Phys. Rev. D 15 (1977) 1958 [INSPIRE].

[11] R.S. Chivukula and H. Georgi, Composite technicolor standard model, Phys. Lett. B 188 (1987) 99 [INSPIRE].

[12] A.J. Buras, P. Gambino, M. Gorbahn, S. Jager and L. Silvestrini, Universal unitarity triangle and physics beyond the standard model, Phys. Lett. B 500 (2001) 161 [hep-ph/0007085] [INSPIRE].

[13] G. D'Ambrosio, G.F. Giudice, G. Isidori and A. Strumia, Minimal flavor violation: an effective field theory approach, Nucl. Phys. B 645 (2002) 155 [hep-ph/0207036] [INSPIRE].

[14] A.J. Buras, M.V. Carlucci, S. Gori and G. Isidori, Higgs-mediated FCNCs: natural flavour conservation vs. minimal flavour violation, JHEP 10 (2010) 009 [arXiv: 1005.5310] [INSPIRE].

[15] G. Isidori and D.M. Straub, Minimal flavour violation and beyond, Eur. Phys. J. C 72 (2012) 2103 [arXiv: 1202.0464] [INSPIRE]. 
[16] E. Cervero and J.-M. Gerard, Minimal violation of flavour and custodial symmetries in a vectophobic two-Higgs-doublet-model, Phys. Lett. B 712 (2012) 255 [arXiv:1202.1973] [INSPIRE].

[17] T.P. Cheng and M. Sher, Mass matrix ansatz and flavor nonconservation in models with multiple Higgs doublets, Phys. Rev. D 35 (1987) 3484 [InSPIRE].

[18] W.-S. Hou, Tree level $t \rightarrow c h$ or $h \rightarrow t \bar{c}$ decays, Phys. Lett. B 296 (1992) 179 [inSPIRE].

[19] A. Cordero-Cid, M.A. Perez, G. Tavares-Velasco and J.J. Toscano, Effective Lagrangian approach to Higgs-mediated FCNC top quark decays, Phys. Rev. D 70 (2004) 074003 [hep-ph/0407127] [INSPIRE].

[20] F. Larios, R. Martinez and M.A. Perez, New physics effects in the flavor-changing neutral couplings of the top quark, Int. J. Mod. Phys. A 21 (2006) 3473 [hep-ph/0605003] [INSPIRE].

[21] P.M. Ferreira, R.B. Guedes and R. Santos, Combined effects of strong and electroweak FCNC effective operators in top quark physics at the CERN LHC, Phys. Rev. D 77 (2008) 114008 [arXiv:0802.2075] [INSPIRE].

[22] J.I. Aranda, A. Cordero-Cid, F. Ramirez-Zavaleta, J.J. Toscano and E.S. Tututi, Higgs mediated flavor violating top quark decays $t \rightarrow u_{i} H, u_{i} \gamma, u_{i} \gamma \gamma$ and the process $\gamma \gamma \rightarrow$ tc in effective theories, Phys. Rev. D 81 (2010) 077701 [arXiv:0911.2304] [InSPIRE].

[23] A. Crivellin, C. Greub and A. Kokulu, Explaining $B \rightarrow D \tau \nu, B \rightarrow D^{*} \tau \nu$ and $B \rightarrow \tau \nu$ in a 2HDM of type-III, Phys. Rev. D 86 (2012) 054014 [arXiv:1206.2634] [InSPIRE].

[24] K.-F. Chen, W.-S. Hou, C. Kao and M. Kohda, When the Higgs meets the top: search for $t \rightarrow c h^{0}$ at the LHC, Phys. Lett. B 725 (2013) 378 [arXiv:1304.8037] [INSPIRE].

[25] BABAR collaboration, J.P. Lees et al., Evidence for an excess of $\bar{B} \rightarrow D^{(*)} \tau^{-} \bar{\nu}_{\tau}$ decays, Phys. Rev. Lett. 109 (2012) 101802 [arXiv:1205.5442] [InSPIRE].

[26] R.S. Gupta and J.D. Wells, Next generation Higgs bosons: theory, constraints and discovery prospects at the Large Hadron Collider, Phys. Rev. D 81 (2010) 055012 [arXiv:0912.0267] [INSPIRE].

[27] D. Atwood, L. Reina and A. Soni, Phenomenology of two Higgs doublet models with flavor changing neutral currents, Phys. Rev. D 55 (1997) 3156 [hep-ph/9609279] [INSPIRE].

[28] P.J. Fox, Z. Ligeti, M. Papucci, G. Perez and M.D. Schwartz, Deciphering top flavor violation at the LHC with B factories, Phys. Rev. D 78 (2008) 054008 [arXiv:0704.1482] [INSPIRE].

[29] F. Mahmoudi and O. Stal, Flavor constraints on the two-Higgs-doublet model with general Yukawa couplings, Phys. Rev. D 81 (2010) 035016 [arXiv:0907.1791] [InSPIRE].

[30] G. Bélanger, B. Dumont, U. Ellwanger, J.F. Gunion and S. Kraml, Global fit to Higgs signal strengths and couplings and implications for extended Higgs sectors, Phys. Rev. D 88 (2013) 075008 [arXiv: 1306.2941] [INSPIRE].

[31] K. Cheung, J.S. Lee and P.-Y. Tseng, Higgs precision (higgcision) era begins, JHEP 05 (2013) 134 [arXiv:1302.3794] [INSPIRE].

[32] C.-W. Chiang and K. Yagyu, Implications of Higgs boson search data on the two-Higgs doublet models with a softly broken $Z_{2}$ symmetry, JHEP 07 (2013) 160 [arXiv:1303.0168] [INSPIRE].

[33] B. Grinstein and P. Uttayarat, Carving out parameter space in type-II two Higgs doublets model, JHEP 06 (2013) 094 [Erratum ibid. 09 (2013) 110] [arXiv: 1304.0028] [INSPIRE]. 
[34] O. Eberhardt, U. Nierste and M. Wiebusch, Status of the two-Higgs-doublet model of type-II, JHEP 07 (2013) 118 [arXiv: 1305.1649] [INSPIRE].

[35] A. Celis, V. Ilisie and A. Pich, LHC constraints on two-Higgs doublet models, JHEP 07 (2013) 053 [arXiv: 1302.4022] [InSPIRE].

[36] S. Chang, S.K. Kang, J.-P. Lee, K.Y. Lee, S.C. Park and J. Song, Comprehensive study of two Higgs doublet model in light of the new boson with mass around $125 \mathrm{GeV}$, JHEP 05 (2013) 075 [arXiv: 1210.3439] [INSPIRE].

[37] S. Chang, S.K. Kang, J.-P. Lee, K.Y. Lee, S.C. Park and J. Song, Two Higgs doublet models for the LHC Higgs boson data at $\sqrt{s}=7$ and 8 TeV, JHEP 09 (2014) 101 [arXiv: 1310.3374] [INSPIRE].

[38] J. Charles et al., Current status of the standard model CKM fit and constraints on $\Delta F=2$ new physics, Phys. Rev. D 91 (2015) 073007 [arXiv: 1501.05013] [INSPIRE].

[39] CKMfitTer Group collaboration, J. Charles et al., CP violation and the CKM matrix: Assessing the impact of the asymmetric B factories, Eur. Phys. J. C 41 (2005) 1 [hep-ph/0406184] [INSPIRE].

[40] N. Craig, J. Galloway and S. Thomas, Searching for signs of the second Higgs doublet, arXiv: 1305.2424 [INSPIRE].

[41] A. Celis, V. Ilisie and A. Pich, Towards a general analysis of LHC data within two-Higgs-doublet models, JHEP 12 (2013) 095 [arXiv: 1310.7941] [INSPIRE].

[42] X.-D. Cheng, Y.-D. Yang and X.-B. Yuan, Phenomenological discriminations of the Yukawa interactions in two-Higgs doublet models with $Z_{2}$ symmetry, Eur. Phys. J. C 74 (2014) 3081 [arXiv: 1401.6657] [INSPIRE].

[43] J. Song and Y.W. Yoon, Gigantic diphoton rate of heavy Higgs bosons in the aligned two Higgs doublet models with small $\tan \beta$, Phys. Rev. D 91 (2015) 113012 [arXiv:1412.5610] [INSPIRE].

[44] J. Bernon, J.F. Gunion, Y. Jiang and S. Kraml, Light Higgs bosons in two-Higgs-doublet models, Phys. Rev. D 91 (2015) 075019 [arXiv:1412.3385] [INSPIRE].

[45] S. Chang, S.K. Kang, J.-P. Lee and J. Song, Higgs potential and hidden light Higgs scenario in two Higgs doublet models, Phys. Rev. D 92 (2015) 075023 [arXiv: 1507.03618] [INSPIRE].

[46] ALEPH collaboration, A. Heister et al., Search for charged Higgs bosons in $e^{+} e^{-}$collisions at energies up to $\sqrt{s}=209 \mathrm{GeV}$, Phys. Lett. B 543 (2002) 1 [hep-ex/0207054] [INSPIRE].

[47] CDF collaboration, A. Abulencia et al., Search for charged Higgs bosons from top quark

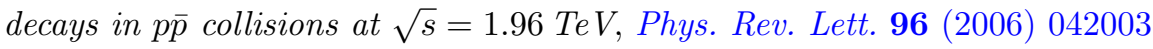
[hep-ex/0510065] [INSPIRE].

[48] D0 collaboration, V.M. Abazov et al., Search for charged Higgs bosons in top quark decays, Phys. Lett. B 682 (2009) 278 [arXiv:0908.1811] [InSPIRE].

[49] CMS collaboration, Search for a light charged Higgs boson in top quark decays in pp collisions at $\sqrt{s}=7 \mathrm{TeV}$, JHEP 07 (2012) 143 [arXiv:1205.5736] [INSPIRE].

[50] ATLAS collaboration, Search for charged Higgs bosons in the $\tau+$ jets final state with $p p$ collision data recorded at $\sqrt{s}=8 \mathrm{TeV}$ with the ATLAS experiment, ATLAS-CONF-2013-090 (2013).

[51] Delphi, OPAl, AlePh, LeP Working Group for Higgs Boson Searches, L3 collaboration, S. Schael et al., Search for neutral MSSM Higgs bosons at LEP, Eur. Phys. J. C 47 (2006) 547 [hep-ex/0602042] [INSPIRE]. 
[52] M. Jung, X.-Q. Li and A. Pich, Exclusive radiative B-meson decays within the aligned two-Higgs-doublet model, JHEP 10 (2012) 063 [arXiv: 1208.1251] [INSPIRE].

[53] Heavy Flavor Averaging Group (HFAG) collaboration, Y. Amhis et al., Averages of b-hadron, c-hadron and $\tau$-lepton properties as of summer 2014, arXiv:1412.7515 [INSPIRE].

[54] Heavy Flavor Averaging Group (HFAG) collaboration, Y. Amhis et al., Rare decays and exotic states in quark flavour physics, talk given at EPS-HEP 2015, July 22-29, Vienna, Austria (2015).

[55] ATLAS collaboration, Search for single top-quark production via FCNC in strong interaction in $\sqrt{s}=8 \mathrm{TeV}$ ATLAS data, ATLAS-CONF-2013-063 (2013).

[56] ATLAS collaboration, Analysis of events with b-jets and a pair of leptons of the same charge in pp collisions at $\sqrt{s}=8 \mathrm{TeV}$ with the ATLAS detector, JHEP 10 (2015) 150 [arXiv:1504.04605] [INSPIRE].

[57] Tevatron Electroweak Working Group, CDF, SLD Heavy Flavor Group, DELPHI, ALEPH, SLD ELECTROWEAK Working Group, LEP Electroweak Working Group, SLD, OPAL, D0, L3 collaboration, J. Alcaraz, Precision electroweak measurements and constraints on the standard model, arXiv:0911.2604 [INSPIRE].

[58] Particle Data Group collaboration, K.A. Olive et al., Review of particle physics, Chin. Phys. C 38 (2014) 090001 [InSPIRE].

[59] Z.-x. Heng, G.-r. Lu, L. Wu and J.M. Yang, Top quark three-body decays in R-violating MSSM, Phys. Rev. D 79 (2009) 094029 [arXiv:0904.0597] [INSPIRE].

[60] G.R. Lu and L. Wu, Flavor-changing neutral-current production of $t$ anti-c in association with a neutral top-Higgs at LHC in the topcolor-assisted technicolor model, Chin. Phys. Lett. 27 (2010) 031401 [INSPIRE].

[61] S. Khatibi and M.M. Najafabadi, Probing the anomalous FCNC interactions in top-higgs final state and charge ratio approach, Phys. Rev. D 89 (2014) 054011 [arXiv:1402.3073] [INSPIRE].

[62] A. Greljo, J.F. Kamenik and J. Kopp, Disentangling flavor violation in the top-Higgs sector at the LHC, JHEP 07 (2014) 046 [arXiv: 1404.1278] [INSPIRE].

[63] L. Wu, Enhancing thj production from top-Higgs FCNC couplings, JHEP 02 (2015) 061 [arXiv: 1407.6113] [INSPIRE].

[64] H. Hesari, H. Khanpour and M.M. Najafabadi, Direct and indirect searches for top-Higgs FCNC couplings, arXiv:1508.07579 [INSPIRE].

[65] CMS collaboration, Combined multilepton and diphoton limit on $t \rightarrow c H$, CMS-PAS-HIG-13-034 (2013).

[66] ATLAS collaboration, Search for top quark decays $t \rightarrow q H$ with $H \rightarrow \gamma \gamma$ using the ATLAS detector, JHEP 06 (2014) 008 [arXiv:1403.6293] [InSPIRE].

[67] CDF collaboration, F. Abe et al., Search for flavor-changing neutral current decays of the top quark in $p \bar{p}$ collisions at $\sqrt{s}=1.8$ TeV, Phys. Rev. Lett. 80 (1998) 2525 [INSPIRE].

[68] CDF collaboration, T. Aaltonen et al., Search for the flavor changing neutral current decay $t \rightarrow Z q$ in pp collisions at $\sqrt{s}=1.96$ TeV, Phys. Rev. Lett. 101 (2008) 192002 [arXiv:0805.2109] [INSPIRE].

[69] D0 collaboration, V.M. Abazov et al., Search for flavor changing neutral currents in decays of top quarks, Phys. Lett. B 701 (2011) 313 [arXiv:1103.4574] [INSPIRE]. 
[70] ATLAS collaboration, A search for flavour changing neutral currents in top-quark decays in pp collision data collected with the ATLAS detector at $\sqrt{s}=7$ TeV, JHEP 09 (2012) 139 [arXiv:1206.0257] [INSPIRE].

[71] CMS collaboration, Search for flavor changing neutral currents in top quark decays in pp collisions at $7 \mathrm{TeV}$, Phys. Lett. B 718 (2013) 1252 [arXiv:1208.0957] [INSPIRE].

[72] CMS collaboration, Search for flavor-changing neutral currents in top-quark decays $t \rightarrow Z q$ in pp collisions at $\sqrt{s}=8 \mathrm{TeV}$, Phys. Rev. Lett. 112 (2014) 171802 [arXiv:1312.4194] [INSPIRE].

[73] CMS collaboration, Search for anomalous single top quark production in association with a photon, CMS-PAS-TOP-14-003 (2014).

[74] CDF collaboration, T. Aaltonen et al., Search for top-quark production via flavor-changing neutral currents in $W+1$ jet events at CDF, Phys. Rev. Lett. 102 (2009) 151801 [arXiv: 0812.3400] [INSPIRE].

[75] D0 collaboration, V.M. Abazov et al., Search for flavor changing neutral currents via quark-gluon couplings in single top quark production using $2.3 \mathrm{fb}^{-1}$ of p $\bar{p}$ collisions, Phys. Lett. B 693 (2010) 81 [arXiv:1006.3575] [INSPIRE].

[76] ATLAS collaboration, Search for single top-quark production via FCNC in strong interaction in $\sqrt{s}=8 \mathrm{TeV}$ ATLAS data, ATLAS-CONF-2013-063 (2013).

[77] CMS collaboration, Search for flavour changing neutral currents in single top events, CMS-PAS-TOP-12-021 (2012).

[78] CMS collaboration, Search for anomalous Wtb couplings and top FCNC in t-channel single-top-quark events, CMS-PAS-TOP-14-007 (2014).

[79] S. Jung, H. Murayama, A. Pierce and J.D. Wells, Top quark forward-backward asymmetry from new t-channel physics, Phys. Rev. D 81 (2010) 015004 [arXiv:0907.4112] [INSPIRE].

[80] J. Cao, L. Wang, L. Wu and J.M. Yang, Top quark forward-backward asymmetry, FCNC decays and like-sign pair production as a joint probe of new physics, Phys. Rev. D 84 (2011) 074001 [arXiv:1101.4456] [INSPIRE].

[81] D0 collaboration, V.M. Abazov et al., First measurement of the forward-backward charge asymmetry in top quark pair production, Phys. Rev. Lett. 100 (2008) 142002 [arXiv: 0712.0851] [INSPIRE].

[82] CDF collaboration, T. Aaltonen et al., Forward-backward asymmetry in top quark production in p p collisions at sqrts $=1.96$ TeV, Phys. Rev. Lett. 101 (2008) 202001 [arXiv:0806.2472] [INSPIRE].

[83] CDF collaboration, T. Aaltonen et al., Evidence for a mass dependent forward-backward asymmetry in top quark pair production, Phys. Rev. D 83 (2011) 112003 [arXiv:1101.0034] [INSPIRE].

[84] CMS collaboration, Search for same-sign top-quark pair production at $\sqrt{s}=7 \mathrm{TeV}$ and limits on flavour changing neutral currents in the top sector, JHEP 08 (2011) 005 [arXiv: 1106.2142] [INSPIRE].

[85] ATLAS collaboration, Search for same-sign top-quark production and fourth-generation down-type quarks in pp collisions at $\sqrt{s}=7 \mathrm{TeV}$ with the ATLAS detector, JHEP 04 (2012) 069 [arXiv: 1202.5520] [INSPIRE].

[86] J.M. Yang, B.-L. Young and X. Zhang, Flavor changing top quark decays in r parity violating SUSY, Phys. Rev. D 58 (1998) 055001 [hep-ph/9705341] [INSPIRE]. 
[87] F. del Aguila, J.A. Aguilar-Saavedra and R. Miquel, Constraints on top couplings in models with exotic quarks, Phys. Rev. Lett. 82 (1999) 1628 [hep-ph/9808400] [INSPIRE].

[88] B. Grzadkowski, J.F. Gunion and P. Krawczyk, Neutral current flavor changing decays for the $Z$ boson and the top quark in two Higgs doublet models, Phys. Lett. B 268 (1991) 106 [INSPIRE].

[89] G. Abbas, A. Celis, X.-Q. Li, J. Lu and A. Pich, Flavour-changing top decays in the aligned two-Higgs-doublet model, JHEP 06 (2015) 005 [arXiv: 1503.06423] [INSPIRE].

[90] J.A. Aguilar-Saavedra and M. Pérez-Victoria, No like-sign tops at Tevatron: constraints on

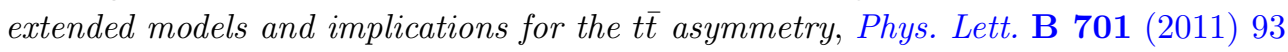
[arXiv:1104.1385] [INSPIRE].

[91] J.A. Aguilar-Saavedra, Effective four-fermion operators in top physics: A Roadmap, Nucl. Phys. B $\mathbf{8 4 3}$ (2011) 638 [Erratum ibid. B 851 (2011) 443] [arXiv: 1008.3562] [INSPIRE].

[92] R. Goldouzian, Search for top quark flavor changing neutral currents in same-sign top quark production, Phys. Rev. D 91 (2015) 014022 [arXiv:1408.0493] [INSPIRE].

[93] A.D. Martin, W.J. Stirling, R.S. Thorne and G. Watt, Parton distributions for the LHC, Eur. Phys. J. C 63 (2009) 189 [arXiv:0901.0002] [InSPIRE].

[94] CMS collaboration, Search for new physics in events with same-sign dileptons and jets in pp collisions at $\sqrt{s}=8 \mathrm{TeV}$, JHEP 01 (2014) 163 [Erratum ibid. 1501 (2015) 014] [arXiv: 1311.6736] [INSPIRE].

[95] P. Ko, Y. Omura and C. Yu, A Resolution of the flavor problem of two Higgs doublet models with an extra $\mathrm{U}(1)_{H}$ symmetry for Higgs flavor, Phys. Lett. B 717 (2012) 202 [arXiv:1204.4588] [INSPIRE].

[96] P. Ko, Y. Omura and C. Yu, $B \rightarrow D^{(*)} \tau \nu$ and $B \rightarrow \tau \nu$ in chiral $\mathrm{U}(1)^{\prime}$ models with flavored multi Higgs doublets, JHEP 03 (2013) 151 [arXiv:1212.4607] [INSPIRE].

[97] M. Freytsis, Z. Ligeti and J.T. Ruderman, Flavor models for $\bar{B} \rightarrow D^{(*)} \tau \bar{\nu}$, Phys. Rev. D 92 (2015) 054018 [arXiv: 1506.08896] [inSPIRE].

[98] G. Cvetič, C.S. Kim, Y.J. Kwon and Y.M. Yook, Decay of $B^{ \pm} \rightarrow \tau^{ \pm}+$"missing momentum" and direct measurement of the mixing parameter $U_{\tau N}$, arXiv:1507.03822 [INSPIRE].

[99] A.G. Akeroyd and S. Recksiegel, The effect of $H^{ \pm}$on $B^{ \pm} \rightarrow \tau^{ \pm} \nu(\tau)$ and $B^{ \pm} \rightarrow \mu^{ \pm}$muon neutrino, J. Phys. G 29 (2003) 2311 [hep-ph/0306037] [INSPIRE].

[100] S. Fajfer, J.F. Kamenik and I. Nisandzic, On the $B \rightarrow D^{*} \tau \bar{\nu}_{\tau}$ sensitivity to new physics, Phys. Rev. D 85 (2012) 094025 [arXiv: 1203. 2654] [InSPIRE].

[101] Y. Sakaki and H. Tanaka, Constraints on the charged scalar effects using the forward-backward asymmetry on $B \rightarrow D^{(*)} \tau \bar{\nu}_{\tau}$, Phys. Rev. D 87 (2013) 054002 [arXiv: 1205.4908] [INSPIRE].

[102] M. Tanaka, Charged Higgs effects on exclusive semitauonic B decays, Z. Phys. C 67 (1995) 321 [hep-ph/9411405] [INSPIRE].

[103] H. Itoh, S. Komine and Y. Okada, Tauonic B decays in the minimal supersymmetric standard model, Prog. Theor. Phys. 114 (2005) 179 [hep-ph/0409228] [INSPIRE].

[104] U. Nierste, S. Trine and S. Westhoff, Charged-Higgs effects in a new $B \rightarrow D \tau \nu$ differential decay distribution, Phys. Rev. D 78 (2008) 015006 [arXiv:0801.4938] [InSPIRE].

[105] BABAR collaboration, J.P. Lees et al., Measurement of an excess of $\bar{B} \rightarrow D^{(*)} \tau^{-} \bar{\nu}_{\tau}$ decays and implications for charged Higgs bosons, Phys. Rev. D 88 (2013) 072012 [arXiv:1303.0571] [INSPIRE]. 
[106] BeLLE collaboration, M. Huschle et al., Measurement of the branching ratio of $\bar{B} \rightarrow D^{(*)} \tau^{-} \bar{\nu}_{\tau}$ relative to $\bar{B} \rightarrow D^{(*)} \ell^{-} \bar{\nu}_{\ell}$ decays with hadronic tagging at Belle, Phys. Rev. D 92 (2015) 072014 [arXiv: 1507.03233] [InSPIRE].

[107] LHCb collaboration, Measurement of the ratio of branching fractions $\mathcal{B}\left(\bar{B}^{0} \rightarrow D^{*+} \tau^{-} \bar{\nu}_{\tau}\right) / \mathcal{B}\left(\bar{B}^{0} \rightarrow D^{*+} \mu^{-} \bar{\nu}_{\mu}\right)$, Phys. Rev. Lett. 115 (2015) 111803 [arXiv: 1506.08614] [INSPIRE].

[108] G. Buchalla, A.J. Buras and M.E. Lautenbacher, Weak decays beyond leading logarithms, Rev. Mod. Phys. 68 (1996) 1125 [hep-ph/9512380] [INSPIRE].

[109] P. Gambino and M. Misiak, Quark mass effects in $\bar{B} \rightarrow X_{s} \gamma$, Nucl. Phys. B 611 (2001) 338 [hep-ph/0104034] [INSPIRE].

[110] A.J. Buras, Climbing NLO and NNLO summits of weak decays, arXiv:1102.5650 [INSPIRE].

[111] B. Grinstein, R.P. Springer and M.B. Wise, Strong interaction effects in weak radiative $\bar{B}$ meson decay, Nucl. Phys. B 339 (1990) 269 [INSPIRE].

[112] A. Ali and C. Greub, A determination of the CKM matrix element ratio $|V(t s)| /|V(c b)|$ from the rare $B$ decays $B \rightarrow K^{*}+\gamma$ and $B \rightarrow X_{s}+\gamma$, Z. Phys. C 60 (1993) 433 [inSPIRE].

[113] M. Misiak, The $b \rightarrow s e^{+} e^{-}$and $b \rightarrow$ s $\gamma$ decays with next-to-leading logarithmic $Q C D$ corrections, Nucl. Phys. B 393 (1993) 23 [Erratum ibid. B 439 (1995) 461] [InSPIRE].

[114] A.J. Buras, Precise determinations of the CKM matrix from CP asymmetries in $B$ decays and $K(L) \rightarrow \pi^{0}$ neutrino anti-neutrino, Phys. Lett. B 333 (1994) 476 [hep-ph/9405368] [INSPIRE].

[115] G. Cella, G. Curci, G. Ricciardi and A. Vicere, The $b \rightarrow s \gamma$ decay revisited, Phys. Lett. B 325 (1994) 227 [hep-ph/9401254] [INSPIRE].

[116] M. Ciuchini, E. Franco, G. Martinelli, L. Reina and L. Silvestrini, $b \rightarrow s \gamma$ and $b \rightarrow s g: a$ theoretical reappraisal, Phys. Lett. B 334 (1994) 137 [hep-ph/9406239] [INSPIRE].

[117] K. Adel and Y.-P. Yao, Exact $\alpha_{s}$ calculation of $b \rightarrow s+\gamma b \rightarrow s+g$, Phys. Rev. D 49 (1994) 4945 [hep-ph/9308349] [INSPIRE].

[118] C. Greub and T. Hurth, Two loop matching of the dipole operators for $b \rightarrow s \gamma$ and $b \rightarrow s g$, Phys. Rev. D 56 (1997) 2934 [hep-ph/9703349] [InSPIRE].

[119] C. Bobeth, M. Misiak and J. Urban, Matching conditions for $b \rightarrow s \gamma$ and $b \rightarrow s$ gluon in extensions of the standard model, Nucl. Phys. B 567 (2000) 153 [hep-ph/9904413] [INSPIRE].

[120] M. Ciuchini, G. Degrassi, P. Gambino and G.F. Giudice, Next-to-leading QCD corrections to $B \rightarrow X_{s} \gamma$ : standard model and two Higgs doublet model, Nucl. Phys. B 527 (1998) 21 [hep-ph/9710335] [INSPIRE].

[121] F. Borzumati and C. Greub, 2HDMs predictions for $\bar{B} \rightarrow X_{s} \gamma$ in NLO QCD, Phys. Rev. D 58 (1998) 074004 [hep-ph/9802391] [INSPIRE].

[122] T. Hermann, M. Misiak and M. Steinhauser, $\bar{B} \rightarrow X_{s} \gamma$ in the two Higgs doublet model up to next-to-next-to-leading order in QCD, JHEP 11 (2012) 036 [arXiv:1208.2788] [INSPIRE].

[123] K.G. Chetyrkin, M. Misiak and M. Münz, Weak radiative $B$ meson decay beyond leading logarithms, Phys. Lett. B 400 (1997) 206 [Erratum ibid. B 425 (1998) 414] [hep-ph/9612313] [INSPIRE].

[124] K.G. Chetyrkin, M. Misiak and M. Münz, $\beta$-functions and anomalous dimensions up to three loops, Nucl. Phys. B 518 (1998) 473 [hep-ph/9711266] [INSPIRE]. 
[125] P. Gambino, M. Gorbahn and U. Haisch, Anomalous dimension matrix for radiative and rare semileptonic B decays up to three loops, Nucl. Phys. B 673 (2003) 238 [hep-ph/0306079] [INSPIRE].

[126] C. Greub, T. Hurth and D. Wyler, Virtual corrections to the decay $b \rightarrow s+\gamma$, Phys. Lett. B 380 (1996) 385 [hep-ph/9602281] [INSPIRE].

[127] C. Greub, T. Hurth and D. Wyler, Virtual $O\left(\alpha_{s}\right)$ corrections to the inclusive decay $b \rightarrow s \gamma$, Phys. Rev. D 54 (1996) 3350 [hep-ph/9603404] [INSPIRE].

[128] A.J. Buras, A. Czarnecki, M. Misiak and J. Urban, Two loop matrix element of the current current operator in the decay $B \rightarrow X_{s} \gamma$, Nucl. Phys. B 611 (2001) 488 [hep-ph/0105160] [INSPIRE].

[129] A.J. Buras, A. Czarnecki, M. Misiak and J. Urban, Completing the NLO QCD calculation of $\bar{B} \rightarrow X_{s} \gamma$, Nucl. Phys. B 631 (2002) 219 [hep-ph/0203135] [InSPIRE].

[130] M. Misiak et al., Estimate of $B\left(\bar{B} \rightarrow X_{s} \gamma\right)$ at $O\left(\alpha_{s}^{2}\right)$, Phys. Rev. Lett. 98 (2007) 022002 [hep-ph/0609232] [INSPIRE].

[131] M. Misiak and M. Steinhauser, NNLO QCD corrections to the $\bar{B} \rightarrow X_{s} \gamma$ matrix elements using interpolation in $m_{c}$, Nucl. Phys. B 764 (2007) 62 [hep-ph/0609241] [INSPIRE].

[132] M. Benzke, S.J. Lee, M. Neubert and G. Paz, Factorization at subleading power and irreducible uncertainties in $\bar{B} \rightarrow X_{s} \gamma$ decay, JHEP 08 (2010) 099 [arXiv: 1003.5012] [INSPIRE].

[133] M. Misiak et al., Updated NNLO QCD predictions for the weak radiative B-meson decays, Phys. Rev. Lett. 114 (2015) 221801 [arXiv:1503.01789] [INSPIRE].

[134] H. Baer, V. Barger, P. Huang, A. Mustafayev and X. Tata, Radiative natural SUSY with a 125 GeV Higgs boson, Phys. Rev. Lett. 109 (2012) 161802 [arXiv:1207.3343] [InSPIRE].

[135] A.J. Buras, S. Jager and J. Urban, Master formulae for $\Delta F=2 N L O Q C D$ factors in the standard model and beyond, Nucl. Phys. B 605 (2001) 600 [hep-ph/0102316] [INSPIRE].

[136] A.J. Buras, P.H. Chankowski, J. Rosiek and L. Slawianowska, $\Delta M(s) / \Delta M(d), \sin 2 \beta$ and the angle $\gamma$ in the presence of new $\Delta F=2$ operators, Nucl. Phys. B 619 (2001) 434 [hep-ph/0107048] [INSPIRE].

[137] A.J. Buras, M. Jamin and P.H. Weisz, Leading and next-to-leading QCD corrections to $\epsilon$ parameter and $B^{0}-\bar{B}^{0}$ mixing in the presence of a heavy top quark, Nucl. Phys. B 347 (1990) 491 [INSPIRE].

[138] J. Urban, F. Krauss, U. Jentschura and G. Soff, Next-to-leading order QCD corrections for the $B^{0}-\overline{B^{0}}$ mixing with an extended Higgs sector, Nucl. Phys. B 523 (1998) 40 [hep-ph/9710245] [INSPIRE].

[139] M. Ciuchini, E. Franco, V. Lubicz, G. Martinelli, I. Scimemi and L. Silvestrini, Next-to-leading order QCD corrections to $\Delta F=2$ effective Hamiltonians, Nucl. Phys. B 523 (1998) 501 [hep-ph/9711402] [INSPIRE].

[140] M. Ciuchini et al., Delta $M_{K}$ and $\epsilon_{K}$ in SUSY at the next-to-leading order, JHEP 10 (1998) 008 [hep-ph/9808328] [INSPIRE].

[141] A.J. Buras, M. Misiak and J. Urban, Two loop QCD anomalous dimensions of flavor changing four quark operators within and beyond the standard model, Nucl. Phys. B 586 (2000) 397 [hep-ph/0005183] [INSPIRE].

[142] S. Jung and J.D. Wells, Gaugino physics of split supersymmetry spectra at the LHC and future proton colliders, Phys. Rev. D 89 (2014) 075004 [arXiv: 1312.1802] [INSPIRE]. 
[143] A. Djouadi, L. Maiani, A. Polosa, J. Quevillon and V. Riquer, Fully covering the MSSM Higgs sector at the LHC, JHEP 06 (2015) 168 [arXiv: 1502.05653] [INSPIRE].

[144] M.E. Luke and M.J. Savage, Flavor changing neutral currents in the Higgs sector and rare top decays, Phys. Lett. B 307 (1993) 387 [hep-ph/9303249] [INSPIRE].

[145] G. Passarino and M.J.G. Veltman, One loop corrections for $e^{+} e^{-}$annihilation into $\mu^{+} \mu^{-}$ in the Weinberg model, Nucl. Phys. B 160 (1979) 151 [INSPIRE].

[146] G. 't Hooft and M.J.G. Veltman, Scalar one loop integrals, Nucl. Phys. B 153 (1979) 365 [INSPIRE].

[147] T. Hahn and M. Pérez-Victoria, Automatized one loop calculations in four-dimensions and D-dimensions, Comput. Phys. Commun. 118 (1999) 153 [hep-ph/9807565] [INSPIRE]. 